\title{
Proximal forward-backward splitting method for zeros of sum accretive operators for a fixed point set and inverse problems
}

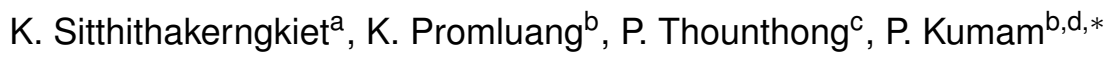 \\ a Nonlinear Dynamic Analysis Research Center, Department of Mathematics, Faculty of Applied Science, King Mongkut's University of \\ Technology North Bangkok (KMUTNB), 1518, Pracharat 1 Road, Wongsawang, Bangsue, Bangkok 10800, Thailand. \\ ${ }^{b}$ KMUTTFixed Point Research Laboratory, Department of Mathematics, Room SCL 802 Fixed Point Laboratory, Science Laboratory \\ Building, Faculty of Science, King Mongkut's University of Technology Thonburi (KMUTT), 126, Pracha-Uthit Road, Bang Mod, Thung \\ Khru, Bangkok 10140, Thailand. \\ ${ }^{c}$ Renewable Energy Research Centre \& Department of Teacher Training in Electrical Engineering, Faculty of Technical Education, King \\ Mongkut's University of Technology North Bangkok (KMUTNB), Wongsawang, Bangsue, Bangkok 10800, Thailand. \\ ${ }^{d}$ KMUTT-Fixed Point Theory and Applications Research Group, Theoretical and Computational Science Center (TaCS), Science \\ Laboratory Building, Faculty of Science, King Mongkut's University of Technology Thonburi (KMUTT), 126, Pracha-Uthit Road, Bang \\ Mod, Thung Khru, Bangkok 10140, Thailand.
}

\begin{abstract}
In this paper, we investigate regularization method via a proximal point algorithm for solving treating sum of two accretive operators and fixed point problems. Strong convergence theorems are established in the framework of Banach spaces. Also we apply our result to variational inequalities and equilibrium problems. Furthermore, an illustrative numerical example is presented. (C) 2017 All rights reserved.
\end{abstract}

Keywords: Regularization method, proximal point algorithm, zero points, accretive operators, inverse problems. 2010 MSC: 47H09, 47H17, 47J25, 49J40.

\section{Introduction}

Many important problems have reformulation which require finding common zero points of nonlinear operators, for instance, inverse problems, variational inequality, optimization problems and fixed point problems. In this paper, we use $A^{-1}(0)$ to denote the set of zeros point of $A$, where $A$ is a maximal monotone operator. A well-known method for solving zero points of maximal monotone operators is the proximal point algorithm (PPA). First, Martinet [13] introduced the PPA in a Hilbert space $H$, that is, for starting $x_{0} \in H$, a sequence $\left\{x_{n}\right\}$ generated by

$$
x_{n+1}=J_{r_{n}}^{A}\left(x_{n}\right), \quad \forall n \in \mathbb{N},
$$

where $A$ is a maximal monotone operator, $J_{r_{n}}^{A}=\left(I+r_{n} A\right)^{-1}$ is the resolvent operator of $A$ and $\left\{r_{n}\right\} \subset$

\footnotetext{
${ }^{*}$ Corresponding author

Email addresses: kanokwan.s@sci.kmutnb.ac.th (K. Sitthithakerngkiet), k.promluang@gmail.com (K. Promluang), phtt@kmutnb.ac.th (P. Thounthong), poom. kum@kmutt.ac.th (P. Kumam)
}

doi:10.22436/jmcs.017.04.06 
$(0, \infty)$ is a regularization sequence. An iterative sequence (1.1) is equivalent to

$$
x_{\mathrm{n}} \in x_{\mathrm{n}+1}+\mathrm{r}_{\mathrm{n}} A x_{\mathrm{n}+1}, \quad \forall \mathrm{n} \in \mathbb{N} .
$$

If $\phi: H \rightarrow \mathbb{R} \cup\{\infty\}$ is a proper convex and lower semicontinuous function, then $\mathrm{J}_{\mathrm{r}_{n}}^{A}$ is reduced to

$$
x_{n+1}=\operatorname{argmin}\left\{\phi(y)+\frac{1}{2 r_{n}}\left\|x_{n}-y\right\|^{2}, y \in H\right\}, \forall n \in \mathbb{N} .
$$

Later, Rockafellar [16] studied the proximal point algorithm in framework of a Hilbert space and he also proved that if $\liminf _{n \rightarrow \infty} r_{n}>0$ and $A^{-1}(0) \neq \emptyset$, then the sequence $\left\{x_{n}\right\}$ converges weakly to a solution of a zero point of $A$. Rockafellar [16] has given a more practical method which is an inexact variant of the method as follows

$$
x_{\mathrm{n}+1}=\mathrm{J}_{\mathrm{r}_{\mathrm{n}}}^{\mathrm{A}} \mathrm{x}_{\mathrm{n}}+e_{\mathrm{n}}, \quad \forall \mathrm{n} \in \mathbb{N},
$$

where $\left\{e_{n}\right\}$ is an error sequence. It was shown that if $e_{n} \rightarrow 0$ quickly enough such that $\sum_{n=1}^{\infty}\left\|e_{n}\right\|<\infty$, then $x_{n} \rightarrow z \in H$, with $0 \in A(z)$.

On the other hand, the Tikhonov method which generates a sequence $\left\{\tilde{x_{n}}\right\}$ defined by

$$
\tilde{x_{\mathrm{n}}}=\mathrm{J}_{\mathrm{r}_{\mathrm{n}}}^{\mathrm{A}} \mathrm{u}, \quad \forall \mathrm{n} \in \mathbb{N},
$$

where $u \in H$ and $r_{n}>0$ such that $r_{n} \rightarrow \infty$ is studied by several authors (see, e.g., Takahashi [20]). The details of Tikhonov Regularization can be found in [23-25].

In 1996, Lehdili and Moudafi [9] combined the technique of the proximal map and the Tikhonov regularization to introduce the prox-Tikhonov method which generates the sequence $\left\{x_{n}\right\}$ by the algorithm

$$
x_{n+1}=J_{\lambda_{n}}^{A_{n}} x_{n}, \quad \forall n \in \mathbb{N}
$$

where $A_{n}=r_{n} I+A, r_{n}>0$ is viewed as a Tikhonov regularization of $A$. Using the technique of variational distance, Lehdili and Moudafi [9] were able to prove strong convergence of the algorithm (1.2) for solving the variational inclusion problem when $A$ is maximal monotone operator on $\mathrm{H}$ under certain conditions imposed upon the sequences $\left\{\lambda_{n}\right\}$ and $\left\{r_{n}\right\}$.

In 2011, Sahu and Yao [17] also extended PPA for the zero of an accretive operator in a Banach space which has a uniformly Gâteaux differentiable norm by combining the prox-Tikhonov method and the viscosity approximation method. They introduced the iterative method to define the sequence $\left\{x_{n}\right\}$ as follows:

$$
\begin{aligned}
& x_{n+1}=J_{r_{n}}^{A}\left(\left(1-\alpha_{n}\right) x_{n}+\alpha_{n} f\left(x_{n}\right)\right), \quad \forall n \in \mathbb{N}, \\
& z_{n+1}=J_{r_{n}}^{A}\left(\left(1-\alpha_{n}\right) z_{n}+\alpha_{n} f\left(z_{n}\right)+e_{n}\right), \quad \forall n \in \mathbb{N},
\end{aligned}
$$

where $A$ is an accretive operator such that $A^{-1}(0) \neq \emptyset$ and $f$ is a contractive mapping on $C$ and $\left\{e_{n}\right\}$ is an error sequence. Strong convergence results were established in both algorithms. This is a source of idea about resolvent operator can be approximated by contractions.

In the same year, PPA extended to the case of sum of two monotone operators $A$ and $B$ by using the technique of forward-backward splitting method. Manaka and Takahashi [12] introduced the following iterative scheme in a Hilbert space:

$$
\left\{\begin{array}{l}
x_{1} \in C, \\
\left.x_{n+1}=\alpha_{n} x_{n}+\left(1-\alpha_{n}\right) S\right)_{\lambda_{n}}^{A}\left(I-\lambda_{n} B\right) x_{n}, \quad \forall n \geqslant 1,
\end{array}\right.
$$

where $\left\{\alpha_{n}\right\}$ is a sequence in $(0,1),\left\{\lambda_{n}\right\}$ is a positive sequence, $S: C \rightarrow C$ is a nonexpansive mapping, $A$ is a maximal monotone operator, $B$ is an inverse strongly monotone mapping, and $J_{\lambda_{n}}^{A}=\left(I+\lambda_{n} A\right)^{-1}$ is the 
resolvent of $A$. They proved that a sequence $\left\{x_{n}\right\}$ converges weakly to some point $z \in \operatorname{Fix}(S) \cap(A+B)^{-1}(0)$ provided that the control sequence satisfies some conditions.

In 2012, López et al. [11] used the technique of forward-backward splitting methods for accretive operators in Banach spaces. They considered the following algorithms with errors:

$$
\begin{aligned}
& x_{n+1}=\left(1-\alpha_{n}\right) x_{n}+\alpha_{n} J_{r_{n}}^{A}\left(x_{n}-r_{n}\left(B x_{n}+a_{n}\right)\right)+b_{n}, \\
& x_{n+1}=\alpha_{n} u+\left(1-\alpha_{n}\right) J_{r_{n}}^{A}\left(x_{n}-r_{n}\left(B x_{n}+a_{n}\right)\right)+b_{n},
\end{aligned}
$$

where $u \in E,\left\{a_{n}\right\},\left\{b_{n}\right\} \subset E$ and $J_{\lambda_{n}}^{A}=\left(I+\lambda_{n} A\right)^{-1}$ is the resolvent of $A$. An operator $A$ is a maximal accretive operator and $B$ is an inverse strongly accretive. They proved that the sequences $\left\{x_{n}\right\}$ in equations (1.3) and (1.4) is weakly and strongly convergence, respectively.

In 2014, Cho et al. [5] introduced the following iterative scheme in a Hilbert space:

$$
\left\{\begin{array}{l}
x_{1} \in C, \\
z_{n}=\alpha_{n} f\left(x_{n}\right)+\left(1-\alpha_{n}\right) x_{n}, \\
y_{n}=J_{r_{n}}^{A}\left(z_{n}-r_{n} B z_{n}+e_{n}\right), \\
x_{n+1}=\beta_{n} x_{n}+\left(1-\beta_{n}\right)\left(\gamma_{n} y_{n}+\left(1-\gamma_{n}\right) S y_{n}\right) \text { for all } n \in \mathbb{N},
\end{array}\right.
$$

where $\left\{\alpha_{n}\right\},\left\{\beta_{n}\right\},\left\{\gamma_{n}\right\}$ are sequences in $(0,1),\left\{r_{n}\right\}$ is a positive sequence, $A: C \rightarrow H$ is an inverse strongly monotone mapping, $B$ is a maximal monotone operator, and $J_{\lambda_{n}}^{A}=\left(I+\lambda_{n} A\right)^{-1}$ is the resolvent of $A$. Let $S: C \rightarrow C$ be a strictly pseudo-contractive mapping with $k \in[0,1)$, and $f: C \rightarrow C$ be a contractive mapping. They proved that a sequence $\left\{x_{n}\right\}$ converges strongly to a point $\bar{x} \in \operatorname{Fix}(S) \cap(A+B)^{-1}(0)$ if the control sequence satisfies some restrictions.

Motivated by $[5,11,12,17]$, we are interested in the problems for finding a common element of fixed point of nonexpansive $S$ and element of the (quasi) variational inclusion problem as follows:

$$
\text { Find } x \in C \text { such that } x \in \operatorname{Fix}(S) \cap(A+B)^{-1}(0) \text {, }
$$

where $A$ is a single-valued nonlinear mapping and B is a multi-valued mapping.

The purpose of this paper is to introduce an iterative algorithm which is modify regularization method and uses technique of forward-backward splitting methods for finding a common element of the set solution of nonexpansive $S$ and the set solution of fixed point of the variational inclusion problems, where $A$ is an m-accretive operator and $B$ is an inverse-strongly accretive operator in the framework of Banach space with a uniformly convex and 2-uniformly smooth. Furthermore, an illustrative numerical example is presented.

\section{Preliminaries}

Let $E$ be a Banach space and let $E^{*}$ be its dual. Let $\langle\cdot, \cdot\rangle$ be the pairing between $E$ and $E^{*}$. For all $x \in E$ and $x^{*} \in \mathrm{E}^{*}$, the value of $x^{*}$ at $x$ be denoted by $\left\langle x, x^{*}\right\rangle$. The normalized duality mapping $\mathrm{J}: \mathrm{E} \rightarrow 2^{\mathrm{E}^{*}}$ is defined by $J(x)=\left\{x^{*} \in E^{*}:\left\langle x, x^{*}\right\rangle=\|x\|^{2},\|x\|=\left\|x^{*}\right\|\right\}$, for all $x \in E$. A single-value normalized duality mapping is denoted by $j$, which means a mapping $j: E \rightarrow E^{*}$ such that, for all $u \in E, j(u) \in E^{*}$ satisfying the following:

$$
\langle u, j(u)\rangle=\|u\|\|j(u)\|, \quad\|j(u)\|=\|u\| .
$$

If $\mathrm{E}=\mathrm{H}$ is a Hilbert space, then $\mathrm{J}=\mathrm{I}$, where $\mathrm{I}$ is the identity mapping. If $\mathrm{E}$ is smooth Banach space, then $\mathrm{J}$ is single-valued $j$.

A Banach space $E$ is called an Opial's space if for each sequence $\left\{x_{n}\right\}_{n=0}^{\infty}$ in $E$ such that $\left\{x_{n}\right\}$ converges weakly to some $x$ in $E$, the inequality

$$
\liminf _{n \rightarrow \infty}\left\|x_{n}-x\right\|<\liminf _{n \rightarrow \infty}\left\|x_{n}-y\right\|
$$


holds for all $y \in E$ with $y \neq x$. In fact, for any normed linear space $X$ admits the weakly sequentially continuous duality mapping implies $X$ is Opial space. So, a Banach space with a weakly sequentially continuous duality mapping has the Opial's property; see [7].

The modulus of convexity of $E$ is the function $\delta_{\mathrm{E}}:(0,2] \rightarrow[0,1]$ defined by

$$
\delta(\epsilon):=\inf \left\{1-\left\|\frac{x+y}{2}\right\|:\|x\|=\|y\|=1 ;\|x-y\| \geqslant \epsilon\right\} .
$$

E is said to be uniformly convex if and only if $\delta(\epsilon)>0$, for each $\epsilon \in(0,2]$. It is known that a uniformly convex Banach space is reflexive and strictly convex.

Let $S(E)$ be the unit sphere defined by $S(E)=\{x \in E:\|x\|=1\}$. Then the norm $\|\cdot\|$ of $E$ is said to be Gâteaux differentiable norm, if

$$
\lim _{t \rightarrow 0} \frac{\|x+t y\|-\|x\|}{t}
$$

exists for all $x, y \in S(E)$. In this case, space $E$ is called smooth. A spaces $E$ is said to have a uniformly Gâteaux differentiable norm if for each $y \in S(E)$, the limit (2.1) exist uniformly for all $x \in S(E)$. The norm of $E$ is said to be uniformly smooth if the limit (2.1) is attained uniformly for all $x, y \in S(E)$. It is known that if the norm of $E$ is smooth, then the duality mapping $J$ is single-valued and norm to weak* uniformly continuous on each bounded subset of $E$.

On the other hand, the modulus of smoothness of $E$ is the function $\rho:[0, \infty) \rightarrow[0, \infty)$ defined by

$$
\rho(t)=\sup \left\{\frac{1}{2}(\|x+y\|+\|x-y\|)-1: x, y \in S(E),\|x\|=1,\|y\| \leqslant t\right\} .
$$

A Banach space $E$ is smooth if $\rho_{E}(t)>0$ for all $t>0$. A Banach space $E$ is uniformly smooth if and only if $\lim _{t \rightarrow 0} \frac{\rho(t)}{t}=0$. A Banach space $E$ is said to be $q$-uniformly smooth, if for fixed real number $1<q \leqslant 2$, there exists a constant $c>0$ such that $\rho(t) \leqslant c t^{q}$ for all $t>0$. It is known that every q-uniformly smooth space is smooth. In the case $\rho(t) \leqslant c t^{2}$ for $t>0$, these are 2-uniformly smooth. The examples of uniformly convex and 2-uniformly smooth Banach spaces are $L_{p}, l_{p}$ or Sobolev spaces $W_{m}^{p}$, where $p \geqslant 2$. It is well-known that, Hilbert spaces are 2-uniformly convex and 2-uniformly smooth. We know that if $E$ is a reflexive Banach space, then every bounded sequence in $E$ has a weakly convergent subsequence. Note that all uniformly convex and 2-uniformly smooth Banach spaces are reflexive.

Next, we recall the definitions of some operators as follows.

1. Let $f: E \rightarrow E$ be an operator. Then $f$ is called $k$-contraction if there exists a coefficient $k(0<k<1)$ such that

$$
\|f x-f y\| \leqslant k\|x-y\|, \quad \forall x, y \in E .
$$

2. Let $\mathrm{S}: \mathrm{E} \rightarrow \mathrm{E}$ be an operator. Then $\mathrm{S}$ is called nonexpansive if

$$
\|S x-S y\| \leqslant\|x-y\|, \quad \forall x, y \in E .
$$

3. A set-valued operator $A: D(A) \subseteq E \rightarrow 2^{E}$ is called accretive if there exists $j(x-y) \in J(x-y)$ such that $u \in A(x), v \in A(y)$ and

$$
\langle u-v, j(x-y)\rangle \geqslant 0, \quad \forall x, y \in D(A) .
$$

4. Let $A: D(A) \subseteq E \rightarrow E$ be an operator. Then $A$ is called $\alpha$-inverse-strongly accretive if there exists a constant $\alpha>0$ and $j(x-y) \in J(x-y)$ such that

$$
\langle A x-A y, j(x-y)\rangle \geqslant \alpha\|A x-a y\|^{2}, \quad \forall x, y \in D(A) .
$$

5. A set-valued operator $A: D(A) \subseteq E \rightarrow 2^{E}$ is called m-accretive if $A$ is accretive and $R(I+r A)=E$ for some $r>0$, where $I$ is the identity mapping.

Let $C$ and $D$ be nonempty subsets of a Banach space $E$ such that $C$ is a nonempty closed convex and $D \subset C$, then a mapping $Q: C \rightarrow D$ is said to be sunny if $Q(x+t(x-Q(x)))=Q(x)$ whenever $x+t(x-Q(x)) \in C$ for all $x \in C$ and $t \geqslant 0$. 
A mapping $\mathrm{Q}: \mathrm{C} \rightarrow \mathrm{C}$ is called a retraction if $\mathrm{Q}^{2}=\mathrm{Q}$. Also, if a mapping $\mathrm{Q}$ is a retraction, then we have $\mathrm{Q} z=z$ for all $z$ in the range of $\mathrm{Q}$.

Lemma 2.1 ([15]). Let $\mathrm{E}$ be a smooth Banach space and let $\mathrm{C}$ be a nonempty subset of $\mathrm{E}$. Let $\mathrm{Q}: \mathrm{E} \rightarrow \mathrm{C}$ be a retraction and let $\mathrm{J}$ be the normalized duality mapping on $\mathrm{E}$. Then the following statements are equivalent:

(i) $\mathrm{Q}$ is sunny and nonexpansive;

(ii) $\|Q x-Q y\|^{2} \leqslant\langle x-y, J(Q x-Q y)\rangle, \forall x, y \in E$;

(iii) $\|(x-y)-(Q x-Q y)\|^{2} \leqslant\|x-y\|^{2}-\|Q x-Q y\|^{2}$;

(iv) $\langle x-\mathrm{Q} x, \mathrm{~J}(\mathrm{y}-\mathrm{Qx})\rangle \leqslant 0, \forall x \in \mathrm{E}, \mathrm{y} \in \mathrm{C}$.

Lemma 2.2 ([8]). Let $\mathrm{C}$ be a nonempty closed convex subset of a uniformly convex and uniformly smooth Banach space $\mathrm{E}$ and let $\mathrm{S}$ be a nonexpansive mapping of $\mathrm{C}$ into itself with $\operatorname{Fix}(S) \neq \emptyset$. Then, the set $\operatorname{Fix}(\mathrm{S})$ is a sunny nonexpansive retract of $\mathrm{C}$.

It is well-known that if $\mathrm{E}=\mathrm{H}$ is a Hilbert space, then a sunny nonexpansive retraction $\mathrm{Q}_{\mathrm{C}}$ is coincident with the metric projection $P_{C}$ from $E$ onto $C$, that is $Q_{C}=P_{C}$. Let $C$ be a nonempty closed convex subset of $E$.

In the sequel for the proof of our main results, we also need the following lemmas.

Lemma 2.3 ([18]). Let $\mathrm{E}$ be a Banach space and $\mathrm{J}$ be a normal duality mapping. Then there exists $\mathrm{j}(\mathrm{x}+\mathrm{y}) \in \mathrm{J}(\mathrm{x}+\mathrm{y})$ such that

$$
\|x+y\|^{2} \leqslant\|x\|^{2}+2\langle y, j(x+y)\rangle, \quad j(x+y) \in J(x+y)
$$

for any given $\mathrm{x}, \mathrm{y} \in \mathrm{E}$.

Lemma 2.4 ([11]). Let $\mathrm{E}$ be a real Banach space and let $\mathrm{C}$ be a nonempty closed and convex subset of $\mathrm{E}$. Let $\mathrm{B}: \mathrm{C} \rightarrow \mathrm{E}$ be a single-valued operator and $\alpha$-inverse strongly accretive operator and let $\mathrm{A}$ be an m-accretive operator in $\mathrm{E}$ with $\mathrm{D}(\mathrm{A}) \supset \mathrm{C}$ and $\mathrm{D}(\mathrm{B}) \supset \mathrm{C}$. Then

$$
\operatorname{Fix}\left(J_{r}^{A}(I-r B)\right)=(A+B)^{-1}(0),
$$

where $\mathrm{J}_{\mathrm{r}}^{\mathrm{A}}=(\mathrm{I}+\mathrm{rA})^{-1}$ is a resolvent of $\mathrm{A}$ for all $\mathrm{r}>0$.

Lemma 2.5 (The resolvent identity, [2]). Let $\mathrm{E}$ be a Banach space and A be an m-accretive operator. Then

$$
\mathrm{J}_{\mathrm{r}}^{A} \mathrm{x}=\mathrm{J}_{\mathrm{s}}^{\mathrm{A}}\left(\frac{\mathrm{s}}{\mathrm{r}} \mathrm{x}+\left(1-\frac{\mathrm{s}}{\mathrm{r}}\right) \mathrm{J}_{\mathrm{r}}^{\mathrm{A}} \mathrm{x}\right)
$$

for all $\mathrm{r}>0, \mathrm{~s}>0$ and $\mathrm{x} \in \mathrm{E}$.

Lemma 2.6 ([1]). Let $\mathrm{C}$ be a nonempty closed convex subset of a 2-uniformly smooth Banach space $\mathrm{E}$ with the 2-uniformly smooth constant $\mathrm{K}$ and the mapping $\mathrm{B}: \mathrm{C} \rightarrow \mathrm{E}$ be an $\alpha$-inverse strongly accretive operator. Then, we have

$$
\|(I-r B) x-(I-r B) y\|^{2} \leqslant\|x-y\|^{2}-2 r\left(\alpha-K^{2} r\right)\|B x-B y\|^{2},
$$

where $\mathrm{I}$ is the identity mapping. In particular, if $\mathrm{r} \in\left(0, \frac{\alpha}{\mathrm{K}^{2}}\right)$, then $(\mathrm{I}-\mathrm{rB})$ is nonexpansive.

Lemma 2.7 (Demiclosed principle, [4]). Let $\mathrm{C}$ be a nonempty, closed and convex subset of a uniformly convex Banach space $\mathrm{E}$ and $\mathrm{S}: \mathrm{C} \rightarrow \mathrm{E}$ be a nonexpansive mapping with $\mathrm{Fix}(\mathrm{S}) \neq \emptyset$. Then $\mathrm{I}-\mathrm{S}$ is demiclosed at zero, i.e., $\mathrm{x}_{\mathrm{n}} \rightarrow \mathrm{x}$ and $\mathrm{x}_{\mathrm{n}}-\mathrm{S} \mathrm{x}_{\mathrm{n}} \rightarrow 0$ imply $\mathrm{x}=\mathrm{S} \mathrm{x}$.

Lemma 2.8 ([19]). Let $\left\{x_{n}\right\}$ and $\left\{z_{n}\right\}$ be two bounded sequences in Banach space $E$ and let $\left\{\beta_{n}\right\}$ be a sequence in $[0,1]$ with $0<\liminf _{n \rightarrow \infty} \beta_{n} \leqslant \limsup _{n \rightarrow \infty} \beta_{n}<1$. Suppose $x_{n+1}=\left(1-\beta_{n}\right) z_{n}+\beta_{n} x_{n}$ for all integers $\mathrm{n} \geqslant 0$ and $\lim \sup _{n \rightarrow \infty}\left(\left\|z_{n+1}-z_{n}\right\|-\left\|x_{n+1}-x_{n}\right\|\right) \leqslant 0$. Then $\lim _{n \rightarrow \infty}\left\|z_{n}-x_{n}\right\|=0$.

Lemma 2.9 ([10]). Assume that $\left\{\mathrm{a}_{\mathrm{n}}\right\}$ is a sequence of nonnegative real numbers satisfying the condition

$$
a_{n+1} \leqslant\left(1-t_{n}\right) a_{n}+t_{n} b_{n}+c_{n}, \forall n \geqslant 0,
$$

where $\left\{t_{n}\right\}$ is a number sequence in $(0,1)$ with $\lim _{n \rightarrow \infty} t_{n}=0$ and $\sum_{n \rightarrow \infty}^{\infty} t_{n}=\infty,\left\{b_{n}\right\}$ is a sequence such that $\limsup _{n \rightarrow \infty} b_{n} \leqslant 0$ and $\left\{c_{n}\right\}$ is a positive number sequence with $\sum_{n=0}^{\infty} c_{n}<\infty$. Then, $\lim _{n \rightarrow \infty} a_{n}=0$. 


\section{Main results}

Before proving our main result, we need the following lemma.

Lemma 3.1. Let $\mathrm{E}$ be a uniformly convex and uniformly smooth Banach space. Let $\mathrm{C}$ be a nonempty closed convex subset of $\mathrm{E}$. Let $\mathrm{A}: \mathrm{D}(\mathrm{A}) \subseteq \mathrm{C} \rightarrow 2^{\mathrm{E}}$ be an $\mathrm{m}$-accretive operator and $\mathrm{B}: \mathrm{C} \rightarrow \mathrm{E}$ be an $\alpha$-inverse strongly accretive operator. Let $\mathrm{S}: \mathrm{C} \rightarrow \mathrm{C}$ be a nonexpansive mapping and let $\mathrm{f}: \mathrm{C} \rightarrow \mathrm{C}$ be a contraction mapping with the constant $\mathrm{k} \in(0,1)$. Let $\mathrm{J}_{r_{n}}^{A}=\left(I+r_{n} A\right)^{-1}$ be a resolvent of $A$ for $r_{n}>0$ such that $\operatorname{Fix}(S) \cap(A+B)^{-1}(0) \neq \emptyset$. If we define operator $W_{n}: C \rightarrow C$ by $W_{n}(x):=S J_{r_{n}}^{A}\left(\left(I-r_{n} B\right)\left[\alpha_{n} f x+\left(1-\alpha_{n}\right) x\right]+e_{n}\right)$ for all $x \in C$, where $\alpha_{n} \in(0,1), r_{n}>0$, then $W_{n}$ is a contraction operator and has a unique fixed point.

Proof. Since $S, J_{r_{n}}^{A}$, and $\left(I-r_{n} B\right)$ are nonexpansive, then we know that $W_{n}$ is nonexpansive. Since $f$ is a contraction mapping with coefficient $k \in(0,1)$ we have

$$
\begin{aligned}
\left\|W_{n} x-W_{n} y\right\| & =\left\|S J_{r_{n}}^{A}\left(\left(I-r_{n} B\right)\left[\alpha_{n} f(x)+\left(1-\alpha_{n}\right) x\right]+e_{n}\right)-S J_{r_{n}}^{A}\left(\left(I-r_{n} B\right)\left[\alpha_{n} f(y)+\left(1-\alpha_{n}\right) y\right]+e_{n}\right)\right\| \\
& \leqslant\left\|\left(\left(I-r_{n} B\right)\left[\alpha_{n} f(x)+\left(1-\alpha_{n}\right) x\right]+e_{n}\right)-\left(\left(I-r_{n} B\right)\left[\alpha_{n} f(y)+\left(1-\alpha_{n}\right) y\right]+e_{n}\right)\right\| \\
& \leqslant\left\|\left[\alpha_{n} f(x)+\left(1-\alpha_{n}\right) x\right]-\left[\alpha_{n} f(y)+\left(1-\alpha_{n}\right) y\right]\right\| \\
& =\left\|\alpha_{n}(f(x)-f(y))+\left(1-\alpha_{n}\right)(x-y)\right\| \\
& \leqslant \alpha_{n}\|f(x)-f(y)\|+\left(1-\alpha_{n}\right)\|x-y\| \\
& \leqslant \alpha_{n} k\|x-y\|+\left(1-\alpha_{n}\right)\|x-y\| \\
& =\left(\alpha_{n} k+\left(1-\alpha_{n}\right)\right)\|x-y\| .
\end{aligned}
$$

Since $0<\left(\alpha_{n} k+\left(1-\alpha_{n}\right)\right)<1$, it follows that $W_{n}$ is a contraction mapping of $C$ into itself. By Banach contraction principle, then there exists a unique fixed point, i.e., we say $\bar{x}=W_{n} \bar{x}$. Moreover, using Lemma 2.2, the set $\operatorname{Fix}\left(W_{n}\right)$ is sunny nonexpansive retraction of $C$. Hence there exists a unique fixed point $\bar{x} \in \operatorname{Fix}\left(W_{n}\right)=\operatorname{Fix}(S) \cap(A+B)^{-1}(0):=\Omega$, namely $Q_{\Omega} f(\bar{x})=\bar{x}=W_{n} \bar{x}$.

Theorem 3.2. Let $\mathrm{E}$ be a uniformly convex and 2-uniformly smooth Banach space with weakly sequentially continuous duality mapping. Let $\mathrm{C}$ be a nonempty closed convex subset of $\mathrm{E}$. Let $\mathrm{A}: \mathrm{D}(\mathrm{A}) \subseteq \mathrm{C} \rightarrow 2^{\mathrm{E}}$ be an m-accretive operator and $\mathrm{B}: \mathrm{C} \rightarrow \mathrm{E}$ be an $\alpha$-inverse strongly accretive operator. Let $\mathrm{S}: \mathrm{C} \rightarrow \mathrm{C}$ be a nonexpansive mapping and let $\mathrm{f}: \mathrm{C} \rightarrow \mathrm{C}$ be a contraction mapping with the constant $\mathrm{k} \in(0,1)$. Let $\mathrm{J}_{\mathrm{r}_{n}}^{\mathrm{A}}=\left(\mathrm{I}+\mathrm{r}_{\mathrm{n}} \mathrm{A}\right)^{-1}$ be a resolvent of $\mathrm{A}$ for $\mathrm{r}_{\mathrm{n}}>0$. Assume that $\operatorname{Fix}(\mathrm{S}) \cap(\mathrm{A}+\mathrm{B})^{-1}(0) \neq \emptyset$.

For given $\mathrm{x}_{0} \in \mathrm{C}$, let $\left\{\mathrm{x}_{\mathrm{n}}\right\}$ be a sequence defined by the following:

$$
\left\{\begin{array}{l}
y_{n}=\alpha_{n} f\left(x_{n}\right)+\left(1-\alpha_{n}\right) x_{n}, \\
x_{n+1}=\beta_{n} x_{n}+\left(1-\beta_{n}\right) S J_{r_{n}}^{A}\left(y_{n}-r_{n} B y_{n}+e_{n}\right), \quad \forall n \geqslant 0,
\end{array}\right.
$$

where $\left\{\alpha_{n}\right\},\left\{\beta_{n}\right\}$ are real number sequences in $(0,1),\left\{r_{n}\right\}$ is a real number sequence in $\left(0, \frac{\alpha}{K^{2}}\right), K>0$ is the 2uniformly smooth constant of $\mathrm{E}$ and $\left\{\mathrm{e}_{\mathrm{n}}\right\}$ is a sequence in $\mathrm{E}$. Assume that the control sequences satisfy the following conditions:

(a) $\lim _{n \rightarrow \infty} \alpha_{n}=0$, and $\sum_{n=1}^{\infty} \alpha_{n}=\infty$;

(b) $0<\liminf _{n \rightarrow \infty} \beta_{n} \leqslant \limsup _{n \rightarrow \infty} \beta_{n}<1$;

(c) $\lim _{n \rightarrow \infty} r_{n}=r$, and $r \in\left(0, \frac{\alpha}{\mathrm{K}^{2}}\right)$;

(d) $\sum_{n=0}^{\infty}\left\|e_{n}\right\|<\infty$.

Then, the sequence $\left\{x_{n}\right\}$ converges strongly to a point $\bar{x} \in \operatorname{Fix}(S) \cap(A+B)^{-1}(0)$, where $\bar{x}=Q_{\Omega} f(\bar{x})$ and $Q_{\Omega} f$ is a sunny nonexpansive retraction from $\mathrm{E}$ onto $\Omega$.

Proof.

Step 1. We want to show that $\left\{x_{n}\right\}$ is bounded.

Fix $p \in \operatorname{Fix}(S) \cap(A+B)^{-1}(0) \neq \emptyset$. So, we have $p \in \operatorname{Fix}(S)$ and $p \in(A+B)^{-1}(0)=\operatorname{Fix}\left(J_{r_{n}}^{A}\left(I-r_{n} B\right)\right)$ 
(see Lemma 2.4). Observe that, we consider

$$
\begin{aligned}
\left\|y_{n}-p\right\| & =\left\|\alpha_{n} f\left(x_{n}\right)+\left(1-\alpha_{n}\right) x_{n}-p\right\| \\
& \leqslant \alpha_{n}\left\|f\left(x_{n}\right)-p\right\|+\left(1-\alpha_{n}\right)\left\|x_{n}-p\right\| \\
& \leqslant \alpha_{n}\left(\left\|f\left(x_{n}\right)-f(p)\right\|+\|f(p)-p\|\right)+\left(1-\alpha_{n}\right)\left\|x_{n}-p\right\| \\
& \leqslant \alpha_{n} k\left\|x_{n}-p\right\|+\alpha_{n}\|f(p)-p\|+\left(1-\alpha_{n}\right)\left\|x_{n}-p\right\| \\
& =\left[\alpha_{n} k+\left(1-\alpha_{n}\right)\right]\left\|x_{n}-p\right\|+\alpha_{n}\|f(p)-p\| \\
& =\left[1-\alpha_{n}(1-k)\right]\left\|x_{n}-p\right\|+\alpha_{n}\|f(p)-p\| .
\end{aligned}
$$

We set $z_{n}:=S J_{r_{n}}^{A}\left(y_{n}-r_{n} B y_{n}+e_{n+1}\right)$. Since $J_{r_{n}}^{A}$ and $I-r_{n} B$ are nonexpansive mappings, and from (3.2), it follows that

$$
\begin{aligned}
\left\|x_{n+1}-p\right\| & =\left\|\beta_{n} x_{n}+\left(1-\beta_{n}\right) z_{n}-p\right\| \\
& \leqslant \beta_{n}\left\|x_{n}-p\right\|+\left(1-\beta_{n}\right)\left\|z_{n}-p\right\| \\
& =\beta_{n}\left\|x_{n}-p\right\|+\left(1-\beta_{n}\right)\left\|S J_{r_{n}}^{A}\left(y_{n}-r_{n} B y_{n}+e_{n}\right)-S p\right\| \\
& \leqslant \beta_{n}\left\|x_{n}-p\right\|+\left(1-\beta_{n}\right)\left\|J_{r_{n}}^{A}\left(y_{n}-r_{n} B y_{n}+e_{n}\right)-p\right\| \\
& =\beta_{n}\left\|x_{n}-p\right\|+\left(1-\beta_{n}\right)\left\|J_{r_{n}}^{A}\left(y_{n}-r_{n} B y_{n}+e_{n}\right)-J_{r_{n}}^{A}\left(I-r_{n} B\right) p\right\| \\
& \leqslant \beta_{n}\left\|x_{n}-p\right\|+\left(1-\beta_{n}\right)\left\|\left(y_{n}-r_{n} B y_{n}+e_{n}\right)-\left(I-r_{n} B\right) p\right\| \\
& =\beta_{n}\left\|x_{n}-p\right\|+\left(1-\beta_{n}\right)\left\|\left(I-r_{n} B\right) y_{n}-\left(I-r_{n} B\right) p+e_{n}\right\| \\
& \leqslant \beta_{n}\left\|x_{n}-p\right\|+\left(1-\beta_{n}\right)\left(\left\|\left(I-r_{n} B\right) y_{n}-\left(I-r_{n} B\right) p\right\|+\left\|e_{n}\right\|\right) \\
& \leqslant \beta_{n}\left\|x_{n}-p\right\|+\left(1-\beta_{n}\right)\left[\left\|y_{n}-p\right\|+\left\|e_{n}\right\|\right] \\
& \leqslant \beta_{n}\left\|x_{n}-p\right\|+\left(1-\beta_{n}\right)\left[\left(1-\alpha_{n}(1-k)\right)\left\|x_{n}-p\right\|+\alpha_{n}\|f(p)-p\|\right]+\left(1-\beta_{n}\right)\left\|e_{n}\right\| \\
& =\beta_{n}\left\|x_{n}-p\right\|+\left[\left(1-\beta_{n}\right)\left(1-\alpha_{n}(1-k)\right)\right]\left\|x_{n}-p\right\|+\left(1-\beta_{n}\right) \alpha_{n}\|f(p)-p\|+\left(1-\beta_{n}\right)\left\|e_{n}\right\| \\
& =\left[\beta_{n}+\left(1-\beta_{n}\right)-\alpha_{n}(1-k)\left(1-\beta_{n}\right)\right]\left\|x_{n}-p\right\|+\left(1-\beta_{n}\right) \alpha_{n}\|f(p)-p\|+\left(1-\beta_{n}\right)\left\|e_{n}\right\| \\
& =\left[1-\left(1-\beta_{n}\right) \alpha_{n}(1-k)\right]\left\|x_{n}-p\right\|+\left(1-\beta_{n}\right) \alpha_{n}\|f(p)-p\|+\left(1-\beta_{n}\right)\left\|e_{n}\right\| \\
& =\left[1-\lambda_{n}(1-k)\right]\left\|x_{n}-p\right\|+\lambda_{n}\|f(p)-p\|+\left\|e_{n}\right\|
\end{aligned}
$$

where $\lambda_{n}:=\left(1-\beta_{n}\right) \alpha_{n}$. Then, it follows that

$$
\begin{aligned}
\left\|x_{n+1}-p\right\| & \leqslant \max \left\{\left\|x_{n}-p\right\|, \frac{\|f(p)-p\|}{1-k}\right\}+\left\|e_{n}\right\| \\
& \leqslant \max \left\{\left\|x_{n-1}-p\right\|, \frac{\|f(p)-p\|}{1-k}\right\}+\left\|e_{n-1}\right\|+\left\|e_{n}\right\| \\
& \leqslant \max \left\{\left\|x_{n-2}-p\right\|, \frac{\|f(p)-p\|}{1-k}\right\}+\left\|e_{n-2}\right\|+\left\|e_{n-1}\right\|+\left\|e_{n}\right\| \\
& \vdots \\
& \leqslant \max \left\{\left\|x_{0}-p\right\|, \frac{\|f(p)-p\|}{1-k}\right\}+\sum_{i=0}^{n}\left\|e_{i}\right\|<\infty .
\end{aligned}
$$

It follows by mathematical induction, we conclude that

$$
\left\|x_{n+1}-p\right\| \leqslant \max \left\{\left\|x_{0}-p\right\|,(1-k)^{-1}\|f(p)-p\|\right\}+\sum_{i=0}^{n}\left\|e_{i}\right\|, \quad \forall n \geqslant 0 .
$$

By condition (d), we get that $\left\{x_{n}\right\}$ is bounded. Since $y_{n}=\alpha_{n} f\left(x_{n}\right)+\left(1-\alpha_{n}\right) x_{n}$, we obtain that

$$
\left\|y_{n}-p\right\|=\left\|\alpha_{n} f\left(x_{n}\right)+\left(1-\alpha_{n}\right) x_{n}-p\right\| \leqslant \alpha_{n}\left\|f\left(x_{n}\right)-p\right\|+\left(1-\alpha_{n}\right)\left\|x_{n}-p\right\| .
$$

By (3.3) and the boundness of $\left\{x_{n}\right\}$, then $\left\{y_{n}\right\}$ and $\left\{z_{n}\right\}$ are also bounded. 
Step 2. We want to show that $\lim _{n \rightarrow \infty}\left\|x_{n+1}-x_{n}\right\|=0$.

By Lemma 2.8, we set $v_{n}:=y_{n}-r_{n} A y_{n}+e_{n}$, then $z_{n}:=S J_{r_{n}}^{B} v_{n}$. It follows that

$$
\begin{aligned}
\left\|z_{n+1}-z_{n}\right\| & =\left\|S J_{r_{n+1}}^{A} v_{n+1}-S J_{r_{n}}^{A} v_{n}\right\| \\
& \leqslant\left\|J_{r_{n+1}}^{A} v_{n+1}-J_{r_{n}}^{A} v_{n}\right\| \\
& \leqslant\left\|J_{r_{n+1}}^{A} v_{n+1}-J_{r_{n+1}}^{A} v_{n}\right\|+\left\|J_{r_{n}+1}^{A} v_{n}-J_{r_{n}}^{A} v_{n}\right\| \\
& \leqslant\left\|v_{n+1}-v_{n}\right\|+\left\|J_{r_{n+1}}^{A} v_{n}-J_{r_{n}}^{A} v_{n}\right\| .
\end{aligned}
$$

Next, we compute $\left\|v_{n+1}-v_{n}\right\|$ that

$$
\begin{aligned}
\left\|v_{n+1}-v_{n}\right\| & =\left\|\left(y_{n+1}-r_{n+1} B y_{n+1}+e_{n+1}\right)-\left(y_{n}-r_{n} B y_{n}+e_{n}\right)\right\| \\
& =\left\|\left(I-r_{n} B\right) y_{n+1}-\left(I-r_{n} B\right) y_{n}+\left(r_{n}-r_{n+1}\right) B y_{n+1}+e_{n+1}-e_{n}\right\| \\
& \leqslant\left\|\left(I-r_{n} B\right) y_{n+1}-\left(I-r_{n} B\right) y_{n}\right\|+\left|r_{n}-r_{n+1}\right|\left\|B y_{n+1}\right\|+\left\|e_{n+1}-e_{n}\right\| \\
& \leqslant\left\|y_{n+1}-y_{n}\right\|+\left|r_{n}-r_{n+1}\right|\left\|B y_{n+1}\right\|+\left\|e_{n+1}\right\|+\left\|e_{n}\right\| .
\end{aligned}
$$

Next, we compute $\left\|y_{n+1}-y_{n}\right\|$ that

$$
\begin{aligned}
\left\|y_{n+1}-y_{n}\right\|= & \left\|\left(\alpha_{n+1} f\left(x_{n+1}\right)+\left(1-\alpha_{n+1}\right)\left(x_{n+1}\right)\right)-\left(\alpha_{n} f\left(x_{n}\right)+\left(1-\alpha_{n}\right) x_{n}\right)\right\| \\
= & \| \alpha_{n+1} f\left(x_{n+1}\right)-\alpha_{n} f\left(x_{n+1}\right)+\alpha_{n} f\left(x_{n+1}\right)+\left(1-\alpha_{n+1}\right) x_{n+1}-\alpha_{n} f\left(x_{n}\right)+\left(1-\alpha_{n+1}\right) x_{n} \\
& -\left(1-\alpha_{n+1}\right) x_{n}-\left(1-\alpha_{n}\right) x_{n} \| \\
= & \|\left(\alpha_{n+1}-\alpha_{n}\right) f\left(x_{n+1}\right)+\alpha_{n}\left(f\left(x_{n+1}\right)-f\left(x_{n}\right)\right)+\left(1-\alpha_{n+1}\right)\left(x_{n+1}-x_{n}\right) \\
& +x_{n}\left(\left(1-\alpha_{n+1}\right)-\left(1-\alpha_{n}\right)\right) \| \\
\leqslant & \mid \alpha_{n+1}-\alpha_{n}\left\|f\left(x_{n+1}\right)-x_{n}\right\|+\alpha_{n}\left\|f\left(x_{n+1}\right)-f\left(x_{n}\right)\right\|+\left(1-\alpha_{n+1}\right)\left\|x_{n+1}-x_{n}\right\| \\
= & \left(1-\alpha_{n+1}\right)\left\|x_{n+1}-x_{n}\right\|+h_{n} \\
\leqslant & \left\|x_{n+1}-x_{n}\right\|+h_{n},
\end{aligned}
$$

where $h_{n}=\left|\alpha_{n+1}-\alpha_{n}\right|\left|f\left(x_{n+1}\right)-x_{n}\right|\left|+\alpha_{n} \| f\left(x_{n+1}\right)-f\left(x_{n}\right)\right| \mid$. Then,

$$
\left\|v_{n+1}-v_{n}\right\| \leqslant\left\|x_{n+1}-x_{n}\right\|+h_{n}+g_{n},
$$

where $g_{n}=\left|r_{n}-r_{n+1}\right|\left\|B y_{n+1}\right\|+\left\|e_{n+1}\right\|+\left\|e_{n}\right\|$.

Next, we compute $\left\|J_{r_{n+1}}^{A} v_{n}-J_{r_{n}}^{A} v_{n}\right\|$ by the resolvent identity (see Lemma 2.5) that

$$
\begin{aligned}
\left\|J_{r_{n+1}}^{A} v_{n}-J_{r_{n}}^{A} v_{n}\right\| & =\left\|J_{r_{n}}^{A}\left(\frac{r_{n}}{r_{n+1}} v_{n}+\left(1-\frac{r_{n}}{r_{n+1}}\right) J_{r_{n+1}}^{A} v_{n}\right)-J_{r_{n}}^{A} v_{n}\right\| \\
& \leqslant\left\|\left(\frac{r_{n}}{r_{n+1}} v_{n}+\left(1-\frac{r_{n}}{r_{n+1}}\right) J_{r_{n+1}}^{A} v_{n}\right)-v_{n}\right\| \\
& =\left\|\left(\frac{r_{n}}{r_{n+1}}-1\right) v_{n}+\left(1-\frac{r_{n}}{r_{n+1}}\right) J_{r_{n+1}}^{A} v_{n}\right\| \\
& =\left\|\left(1-\frac{r_{n}}{r_{n+1}}\right) J_{r_{n+1}}^{A} v_{n}-\left(1-\frac{r_{n}}{r_{n+1}}\right) v_{n}\right\| \\
& =\left\|\frac{r_{n+1}-r_{n}}{r_{n+1}}\left(J_{r_{n+1}}^{A} v_{n}-v_{n}\right)\right\| \\
& \leqslant\left|\frac{r_{n+1}-r_{n}}{r_{n+1}}\right|\left\|J_{r_{n+1}}^{A} v_{n}-v_{n}\right\| .
\end{aligned}
$$

From (3.4), (3.5), and (3.6), we obtain

$$
\left\|z_{n+1}-z_{n}\right\| \leqslant\left\|x_{n+1}-x_{n}\right\|+h_{n}+g_{n}+\left|\frac{r_{n+1}-r_{n}}{r_{n+1}}\right|\left\|J_{r_{n+1}}^{A} v_{n}-v_{n}\right\| .
$$


In view of the conditions (a), (c), and (d), it follows that

$$
\left\|z_{\mathfrak{n}+1}-z_{\mathfrak{n}}\right\|-\left\|x_{\mathfrak{n}+1}-x_{\mathfrak{n}}\right\| \leqslant 0 .
$$

Then, we have

$$
\limsup _{n \rightarrow \infty}\left(\left\|z_{n+1}-z_{n}\right\|-\left\|x_{n+1}-x_{n}\right\|\right) \leqslant 0 .
$$

By Lemma 2.8, we conclude that

$$
\lim _{n \rightarrow \infty}\left\|z_{n}-x_{n}\right\|=0
$$

This implies that $\lim _{n \rightarrow \infty}\left\|S J_{r_{n}}^{A}\left(v_{n}\right)-x_{n}\right\|=0$. From (3.1), we observe that

$$
\left\|x_{n+1}-x_{n}\right\|=\left\|\beta_{n} x_{n}+\left(1-\beta_{n}\right) z_{n}-x_{n}\right\| \leqslant\left(1-\beta_{n}\right)\left\|z_{n}-x_{n}\right\| .
$$

By (3.7), then we conclude that

$$
\lim _{n \rightarrow \infty}\left\|x_{n+1}-x_{n}\right\|=0
$$

Step 3. We will show that $\lim _{n \rightarrow \infty}\left\|B y_{n}-B p\right\|=0, \lim _{n \rightarrow \infty}\left\|J_{r_{n}}^{A}\left(v_{n}\right)-y_{n}\right\|=0$, and $\lim _{n \rightarrow \infty} \| S J_{r_{n}}^{A}\left(v_{n}\right)-$ $\mathrm{J}_{\mathrm{r}_{\mathrm{n}}}^{\mathrm{A}}\left(v_{\mathrm{n}}\right) \|=0$.

Step 3.1. First, we show that $\lim _{n \rightarrow \infty}\left\|B y_{n}-B p\right\|=0$. Notice that

$$
\begin{aligned}
\left\|x_{n+1}-p\right\|^{2}= & \left\|\beta_{n} x_{n}+\left(1-\beta_{n}\right) S J_{r_{n}}^{A} v_{n}-p\right\|^{2} \\
\leqslant & \beta_{n}\left\|x_{n}-p\right\|^{2}+\left(1-\beta_{n}\right)\left\|S J_{r_{n}}^{A} v_{n}-p\right\|^{2} \\
= & \beta_{n}\left\|x_{n}-p\right\|^{2}+\left(1-\beta_{n}\right)\left\|v_{n}-\left(I-r_{n} B\right) p\right\|^{2} \\
= & \beta_{n}\left\|x_{n}-p\right\|^{2}+\left(1-\beta_{n}\right)\left\|\left(y_{n}-r_{n} B y_{n}+e_{n}\right)-\left(I-r_{n} B\right) p\right\|^{2} \\
\leqslant & \beta_{n}\left\|x_{n}-p\right\|^{2}+\left(1-\beta_{n}\right)\left[\left\|\left(I-r_{n} B\right) y_{n}-\left(I-r_{n} B\right) p\right\|^{2}\right. \\
& \left.+2\left\|e_{n}\right\|\left\|\left(I-r_{n} B\right) y_{n}-\left(I-r_{n} B\right) p\right\|\right] \\
\leqslant & \beta_{n}\left\|x_{n}-p\right\|^{2}+\left(1-\beta_{n}\right)\left(\left\|y_{n}-p\right\|^{2}-2 r_{n}\left(\alpha-K^{2} r_{n}\right)\left\|B y_{n}-B p\right\|^{2}\right) \\
& +2\left(1-\beta_{n}\right)\left\|e_{n}\right\|\left\|\left(I-r_{n} B\right) y_{n}-\left(I-r_{n} B\right) p\right\| .
\end{aligned}
$$

Set $p_{n}:=\left(1-\beta_{n}\right) 2\left\|e_{n}\right\|\left\|\left(I-r_{n} B\right) y_{n}-\left(I-r_{n} B\right) p\right\|$, we get

$$
\begin{aligned}
\left\|x_{n+1}-p\right\|^{2} \leqslant & \beta_{n}\left\|x_{n}-p\right\|^{2}+\left(1-\beta_{n}\right)\left(\left\|y_{n}-p\right\|^{2}-2 r_{n}\left(\alpha-K^{2} r_{n}\right)\left\|B y_{n}-B p\right\|^{2}\right)+p_{n} \\
= & \beta_{n}\left\|x_{n}-p\right\|^{2}+\left(1-\beta_{n}\right)\left\|y_{n}-p\right\|^{2}-2 r_{n}\left(\alpha-K^{2} r_{n}\right)\left(1-\beta_{n}\right)\left\|B y_{n}-B p\right\|^{2}+p_{n} \\
= & \beta_{n}\left\|x_{n}-p\right\|^{2}+\left(1-\beta_{n}\right)\left\|\alpha_{n} f\left(x_{n}\right)+\left(1-\alpha_{n}\right) x_{n}-p\right\|^{2} \\
& -2 r_{n}\left(\alpha-K^{2} r_{n}\right)\left(1-\beta_{n}\right)\left\|B y_{n}-B p\right\|^{2}+p_{n} .
\end{aligned}
$$

Set $q_{n}:=2 r_{n}\left(\alpha-K^{2} r_{n}\right)\left(1-\beta_{n}\right)\left\|B y_{n}-B p\right\|^{2}$, we get

$$
\begin{aligned}
\left\|x_{n+1}-p\right\|^{2} & \leqslant \beta_{n}\left\|x_{n}-p\right\|^{2}+\left(1-\beta_{n}\right)\left\|\alpha_{n} f\left(x_{n}\right)+\left(1-\alpha_{n}\right) x_{n}-p\right\|^{2}-q_{n}+p_{n} \\
& \leqslant \beta_{n}\left\|x_{n}-p\right\|^{2}+\left(1-\beta_{n}\right) \alpha_{n}\left\|f\left(x_{n}\right)-p\right\|^{2}+\left(1-\beta_{n}\right)\left(1-\alpha_{n}\right)\left\|x_{n}-p\right\|^{2}-q_{n}+p_{n} \\
& =\left(1-\alpha_{n}\left(1-\beta_{n}\right)\right)\left\|x_{n}-p\right\|^{2}+\left(1-\beta_{n}\right) \alpha_{n}\left\|f\left(x_{n}\right)-p\right\|^{2}-q_{n}+p_{n} .
\end{aligned}
$$

It follows that

$$
2 r_{n}\left(\alpha_{n}-K^{2} r_{n}\right)\left(1-\beta_{n}\right)\left\|B y_{n}-B p\right\|^{2}
$$




$$
\begin{aligned}
& \leqslant\left(1-\alpha_{n}\left(1-\beta_{n}\right)\right)\left\|x_{n}-p\right\|^{2}-\left\|x_{n+1}-p\right\|^{2}+\left(1-\beta_{n}\right) \alpha_{n}\left\|f\left(x_{n}\right)-p\right\|^{2}+p_{n} \\
& \leqslant\left\|x_{n}-p\right\|^{2}-\left\|x_{n+1}-p\right\|^{2}+\left(1-\beta_{n}\right) \alpha_{n}\left\|f\left(x_{n}\right)-p\right\|^{2}+p_{n} \\
& =\left\|\left(x_{n}-p\right)+\left(x_{n+1}-p\right)\right\|\left\|\left(x_{n}-p\right)-\left(x_{n+1}-p\right)\right\|+\left(1-\beta_{n}\right) \alpha_{n}\left\|f\left(x_{n}\right)-p\right\|^{2}+p_{n} \\
& =\left\|\left(x_{n}-p\right)+\left(x_{n+1}-p\right)\right\|\left\|x_{n}-x_{n+1}\right\|+\left(1-\beta_{n}\right) \alpha_{n}\left\|f\left(x_{n}\right)-p\right\|^{2}+p_{n} .
\end{aligned}
$$

In view of the conditions (a), (c), (d), and from (3.8), we conclude that $\lim _{n \rightarrow \infty}\left\|B y_{n}-B p\right\|^{2}=0$. This implies

$$
\lim _{n \rightarrow \infty}\left\|B y_{n}-B p\right\|=0 .
$$

Step 3.2. Second, we will show that $\lim _{n \rightarrow \infty}\left\|J_{r_{n}}^{A}\left(v_{n}\right)-y_{n}\right\|=0$.

We observe that

$$
\begin{aligned}
\left\|J_{r_{n}}^{A}\left(v_{n}\right)-p\right\|^{2} \leqslant & \left\|J_{r_{n}}^{A}\left(v_{n}\right)-p\right\|\left\|\left(y_{n}-r_{n} B y_{n}+e_{n}\right)-\left(p-r_{n} B p\right)\right\| \\
= & \frac{1}{2}\left\{\left\|J_{r_{n}}^{A}\left(v_{n}\right)-p\right\|^{2}+\left\|\left(y_{n}-r_{n} B y_{n}+e_{n}\right)-\left(p-r_{n} B p\right)\right\|^{2}\right. \\
& \left.-\left\|\left(J_{r_{n}}^{A}\left(v_{n}\right)-p\right)-\left(y_{n}-r_{n} B y_{n}+e_{n}\right)+\left(p-r_{n} B p\right)\right\|^{2}\right\} \\
= & \frac{1}{2}\left\{\left\|J_{r_{n}}^{A}\left(v_{n}\right)-p\right\|^{2}+\left\|\left(I-r_{n} B\right) y_{n}-\left(I-r_{n} B\right) p+e_{n}\right\|^{2}\right. \\
& \left.-\left\|J_{r_{n}}^{A}\left(v_{n}\right)-y_{n}-r_{n} B y_{n}-e_{n}-r_{n} B p\right\|^{2}\right\} \\
= & \frac{1}{2}\left\{\left\|J_{r_{n}}^{A}\left(v_{n}\right)-p\right\|^{2}+\left\|\left(I-r_{n} B\right) y_{n}-\left(I-r_{n} B\right) p\right\|^{2}+\bar{p}_{n}\right. \\
& \left.-\left\|\left(J_{r_{n}}^{A}\left(v_{n}\right)-y_{n}-e_{n}\right)-r_{n}\left(B y_{n}-B p\right)\right\|^{2}\right\} \\
\leqslant & \frac{1}{2}\left\{\left\|J_{r_{n}}^{A}\left(v_{n}\right)-p\right\|^{2}+\left\|y_{n}-p\right\|^{2}+\bar{p}_{n}\right. \\
& -\left(\left\|J_{r_{n}}^{A}\left(v_{n}\right)-y_{n}-e_{n}\right\|^{2}-2 r_{n}\left\|B y_{n}-B p\right\|\left\|J_{r_{n}}^{A}\left(v_{n}\right)-y_{n}-e_{n}\right\|\right. \\
& \left.\left.+\left\|r_{n} B y_{n}-r_{n} B p\right\|^{2}\right)\right\} \\
= & \frac{1}{2}\left\{\left\|J_{r_{n}}^{A}\left(v_{n}\right)-p\right\|^{2}+\left\|y_{n}-p\right\|^{2}+\bar{p}_{n}-\left\|J_{r_{n}}^{A}\left(v_{n}\right)-y_{n}-e_{n}\right\|^{2}\right. \\
& \left.+2 r_{n}\left\|B y_{n}-B p\right\|\left\|J_{r_{n}}^{A}\left(v_{n}\right)-y_{n}-e_{n}\right\|-\left\|r_{n} B y_{n}-r_{n} B p\right\|^{2}\right\},
\end{aligned}
$$

where $\bar{p}_{\mathrm{n}}=\frac{p_{\mathrm{n}}}{1-\beta_{\mathrm{n}}}$. It follows that

$$
\begin{aligned}
\left\|J_{r_{n}}^{A}\left(v_{n}\right)-p\right\|^{2} \leqslant & \left\|y_{n}-p\right\|^{2}+\bar{p}_{n}-\left\|J_{r_{n}}^{A}\left(v_{n}\right)-y_{n}-e_{n}\right\|^{2} \\
& +2 r_{n}\left\|B y_{n}-B p\right\|\left\|J_{r_{n}}^{A}\left(v_{n}\right)-y_{n}-e_{n}\right\|-\left\|r_{n} B y_{n}-r_{n} B p\right\|^{2} \\
= & \left\|\alpha_{n} f\left(x_{n}\right)+\left(1-\alpha_{n}\right) x_{n}-p\right\|^{2}-\left\|J_{r_{n}}^{A}\left(v_{n}\right)-y_{n}-e_{n}\right\|^{2} \\
& -\left\|r_{n} B y_{n}-r_{n} B p\right\|^{2}+2 r_{n}\left\|B y_{n}-B p\right\|\left\|J_{r_{n}}^{A}\left(v_{n}\right)-y_{n}-e_{n}\right\|+\bar{p}_{n} \\
\leqslant & \alpha_{n}\left\|f\left(x_{n}\right)-p\right\|^{2}+\left(1-\alpha_{n}\right)\left\|x_{n}-p\right\|^{2}-\left\|J_{r_{n}}^{A}\left(v_{n}\right)-y_{n}+e_{n}\right\|^{2} \\
& -r_{n}\left\|B y_{n}-B p\right\|^{2}+2 r_{n}\left\|B y_{n}-B p\right\|\left\|J_{r_{n}}^{A}\left(v_{n}\right)-y_{n}-e_{n}\right\|+\bar{p}_{n} .
\end{aligned}
$$

From (3.10), this implies that

$$
\begin{aligned}
\left\|x_{n+1}-p\right\|^{2} \leqslant & \beta_{n}\left\|x_{n}-p\right\|^{2}+\left(1-\beta_{n}\right)\left\|S J_{r_{n}}^{A}\left(v_{n}\right)-p\right\|^{2} \\
\leqslant & \beta_{n}\left\|x_{n}-p\right\|^{2}+\left(1-\beta_{n}\right)\left\|J_{r_{n}}^{A}\left(v_{n}\right)-p\right\|^{2} \\
\leqslant & \beta_{n}\left\|x_{n}-p\right\|^{2}+\left(1-\beta_{n}\right)\left\{\alpha_{n}\left\|f\left(x_{n}\right)-p\right\|^{2}+\left(1-\alpha_{n}\right)\left\|x_{n}-p\right\|^{2}\right. \\
& \left.-\left\|J_{r_{n}}^{A}\left(v_{n}\right)-y_{n}+e_{n}\right\|^{2}-r_{n}\left\|B y_{n}-B p\right\|^{2}+2 r_{n}\left\|B y_{n}-B p\right\|\left\|J_{r_{n}}^{A}\left(v_{n}\right)-y_{n}-e_{n}\right\|+p_{n}\right\} \\
= & \beta_{n}\left\|x_{n}-p\right\|^{2}+\left(1-\beta_{n}\right) \alpha_{n}\left\|f\left(x_{n}\right)-p\right\|^{2}-\left(1-\beta_{n}\right)\left\|J_{r_{n}}^{A}\left(v_{n}\right)-y_{n}+e_{n}\right\|^{2}
\end{aligned}
$$




$$
\begin{aligned}
& -\left(1-\beta_{n}\right) r_{n}\left\|B y_{n}-B p\right\|^{2}+\left(1-\beta_{n}\right) 2 r_{n}\left\|B y_{n}-B p\right\|\left\|J_{r_{n}}^{A}\left(v_{n}\right)-y_{n}-e_{n}\right\|+\left(1-\beta_{n}\right) p_{n} \\
\leqslant & \left\|x_{n}-p\right\|^{2}+\alpha_{n}\left\|f\left(x_{n}\right)-p\right\|^{2}-\left(1-\beta_{n}\right)\left\|J_{r_{n}}^{A}\left(v_{n}\right)-y_{n}+e_{n}\right\|^{2} \\
& -r_{n}\left\|B y_{n}-B p\right\|^{2}+2 r_{n}\left\|B y_{n}-B p\right\|\left\|J_{r_{n}}^{A}\left(v_{n}\right)-y_{n}-e_{n}\right\|+p_{n} .
\end{aligned}
$$

It follows that

$$
\begin{aligned}
\left(1-\beta_{n}\right)\left\|J_{r_{n}}^{A}\left(v_{n}\right)-y_{n}+e_{n}\right\|^{2} \leqslant & \left\|x_{n}-p\right\|^{2}-\left\|x_{n+1}-p\right\|^{2}+\alpha_{n}\left\|f\left(x_{n}\right)-p\right\|^{2}-r_{n}\left\|B y_{n}-B p\right\|^{2} \\
& +2 r_{n}\left\|B y_{n}-B p\right\|\left\|J_{r_{n}}^{A}\left(v_{n}\right)-y_{n}-e_{n}\right\|+p_{n} . \\
= & \left\|x_{n}-p\right\|^{2}-\left\|x_{n+1}-p\right\|^{2}+s_{n} \\
= & \left\|\left(x_{n}-p\right)+\left(x_{n+1}-p\right)\right\|\left\|\left(x_{n}-p\right)-\left(x_{n+1}-p\right)\right\|+s_{n} \\
= & \left\|\left(x_{n}-p\right)+\left(x_{n+1}-p\right)\right\|\left\|x_{n}-x_{n+1}\right\|+s_{n},
\end{aligned}
$$

where we set $s_{n}:=\alpha_{n}\left\|f\left(x_{n}\right)-p\right\|^{2}-r_{n}\left\|B y_{n}-B p\right\|^{2}+2 r_{n}\left\|B y_{n}-B p\right\|\left\|J_{r_{n}}^{A}\left(v_{n}\right)-y_{n}-e_{n}\right\|+p_{n}$. From (3.11), in view of the conditions (a), (c), (d), and equations (3.8) and (3.9), we conclude that

$$
\lim _{n \rightarrow \infty}\left\|J_{r_{n}}^{A}\left(v_{n}\right)-y_{n}-e_{n}\right\|=0 \text {. }
$$

This in turn implies that

$$
\lim _{n \rightarrow \infty}\left\|J_{r_{n}}^{A}\left(v_{n}\right)-y_{n}\right\|=0
$$

Step 3.3. Lastly, we will show that $\lim _{n \rightarrow \infty}\left\|S J_{r_{n}}^{A}\left(v_{n}\right)-J_{r_{n}}^{A}\left(v_{n}\right)\right\|=0$.

We see that

$$
\left\|y_{n}-x_{n}\right\|=\left\|\alpha_{n} f\left(x_{n}\right)+\left(1-\alpha_{n}\right) x_{n}-x_{n}\right\|=\alpha_{n}\left\|f\left(x_{n}\right)-x_{n}\right\| .
$$

By condition (a), then

$$
\lim _{n \rightarrow \infty}\left\|y_{n}-x_{n}\right\|=0
$$

Next, from (3.12) and equation (3.14), then we see that

$$
\left\|J_{r_{n}}^{A}\left(v_{n}\right)-y_{n}\right\| \leqslant\left\|J_{r_{n}}^{A}\left(v_{n}\right)-y_{n}\right\|+\left\|y_{n}-x_{n}\right\| .
$$

That is,

$$
\lim _{n \rightarrow \infty}\left\|J_{r_{n}}^{A}\left(v_{n}\right)-x_{n}\right\|=0
$$

From equations (3.7) and (3.15), then we see that

$$
\left\|S J_{r_{n}}^{A}\left(v_{n}\right)-J_{r_{n}}^{A}\left(v_{n}\right)\right\| \leqslant\left\|S J_{r_{n}}^{A}\left(v_{n}\right)-x_{n}\right\|+\left\|x_{n}-J_{r_{n}}^{A}\left(v_{n}\right)\right\| .
$$

That is,

$$
\lim _{n \rightarrow \infty}\left\|S J_{r_{n}}^{A}\left(v_{n}\right)-J_{r_{n}}^{A}\left(v_{n}\right)\right\|=0
$$

Step 4. Since $E$ is a uniformly convex and 2-uniformly smooth Banach space, then $E$ is reflexive Banach space. By reflexive Banach space and from $\left\{x_{n}\right\},\left\{y_{n}\right\}$ being bounded, then it has a weakly convergence subsequence. We may assume that $x_{n_{i}} \rightarrow \hat{x}$. In view of $\lim _{n \rightarrow \infty}\left\|y_{n}-x_{n}\right\|=0$, then there exists a subsequence $\left\{y_{n_{i}}\right\}$ of $\left\{y_{n}\right\}$ which converges weakly to $\hat{x}$. We can say that $\left\{y_{n_{i}}\right\}$ also converges weakly to $\hat{x}$, i.e, $y_{n_{i}} \rightarrow \hat{x}$, without loss of generality. We will show that $\hat{x} \in \operatorname{Fix}(S) \cap(A+B)^{-1}(0)=\Omega$.

(i) First, we want to show that $\hat{x} \in \operatorname{Fix}(S)$. Now, we have $y_{n_{i}} \rightarrow \hat{x}$. Since we know that $\left\{J_{r_{n}}^{A}\left(v_{n}\right)\right\}$ is bounded and from $\lim _{n \rightarrow \infty}\left\|J_{r_{n}}^{A}\left(v_{n}\right)-y_{n}\right\|=0$, then we say that $\left\{J_{r_{n_{i}}}^{A}\left(v_{n_{i}}\right)\right\} \rightarrow \hat{x}$.

From (3.16), we have $\lim _{n \rightarrow \infty}\left\|S J_{r_{n_{i}}}^{A}\left(v_{n_{i}}\right)-J_{r_{n_{i}}}^{A}\left(v_{n_{i}}\right)\right\|=0$. By demiclosed principle, this implies $S \hat{x}=\hat{x}$, namely we prove that $\hat{x} \in \operatorname{Fix}(S)$. 
(ii) Next, we show that $J_{r}^{A}(I-r B) \hat{x}=\hat{x}$. From a Banach space with weakly continuous duality mapping has the Opial's condition, see [7]. Suppose $\hat{x} \neq J_{r}^{A}(I-r B) \hat{x}$. By the Opial's condition and conditions (c), (d), then we have

$$
\begin{aligned}
\liminf _{i \rightarrow \infty}\left\|y_{n_{i}}-\hat{x}\right\| & <\liminf _{i \rightarrow \infty}\left\|y_{n_{i}}-J_{r}^{A}(I-r B) \hat{x}\right\| \\
& \leqslant \liminf _{i \rightarrow \infty}^{A}\left\{\left\|y_{n_{i}}-J_{r_{n_{i}}}^{A}\left(v_{n_{i}}\right)\right\|+\left\|J_{r_{n_{i}}}^{A}\left(v_{n_{i}}\right)-J_{r_{n}}^{A}\left(I-r_{n} B\right) \hat{x}\right\|\right\} \\
& =\liminf _{i \rightarrow \infty}\left\{\left\|y_{n_{i}}-J_{r_{n_{i}}}^{A}\left(v_{n_{i}}\right)\right\|+\left\|J_{r}^{A}\left(v_{n_{i}}\right)-J_{r}^{A}(I-r B) \hat{x}\right\|\right\} \\
& \leqslant \liminf _{i \rightarrow \infty}\left\{\left\|y_{n_{i}}-J_{r_{n_{i}}}^{A}\left(v_{n_{i}}\right)\right\|+\left\|v_{n_{i}}-(I-r B) \hat{x}\right\|\right\} \\
& =\liminf _{i \rightarrow \infty}\left\{\left\|y_{n_{i}}-J_{r_{n_{i}}}^{A}\left(v_{n_{i}}\right)\right\|+\left\|(I-r B) y_{n_{i}}-(I-r B) \hat{x}\right\|+\left\|e_{n_{i}}\right\|\right\} \\
& \leqslant \liminf _{i \rightarrow \infty}\left\{\left\|y_{n_{i}}-J_{r_{n_{i}}}^{A}\left(v_{n_{i}}\right)\right\|+\left\|y_{n_{i}}-\hat{x}\right\|+\left\|e_{n_{i}}\right\|\right\} .
\end{aligned}
$$

By (3.12) and condition (d), hence

$$
\liminf _{i \rightarrow \infty}\left\|y_{n_{i}}-\bar{x}\right\|<\liminf _{i \rightarrow \infty}\left\|y_{n_{i}}-\hat{x}\right\|
$$

This is contradiction. Therefore, $J_{r}^{A}(I-r B) \hat{x}=\hat{x}$. This completes the proof that $\hat{x} \in \operatorname{Fix}(S) \cap(A+B)^{-1}(0)=$ $\Omega$.

Step 5. We define operator $W_{n}: C \rightarrow C$ by $W_{n} x:=S J_{r_{n}}^{A}\left(\left(I-r_{n} B\right)\left[\alpha_{n} f x+\left(1-\alpha_{n}\right) x\right]+e_{n}\right)$ for all $x \in$ $C$, where $\alpha_{n} \in(0,1), r_{n}>0$. From Lemma 3.1, the operator $W_{n}$ is a contraction operator and has a unique fixed point. Moreover, using Lemma 2.2, we know that $\bar{x} \in \operatorname{Fix}\left(W_{n}\right)=\operatorname{Fix}(S) \cap(A+B)^{-1}(0):=\Omega$, namely $Q_{\Omega} f(\bar{x})=\bar{x}=W_{n} \bar{x}$ (that is $\left.\hat{x}=\bar{x}\right)$.

Next, we will show that $\lim \sup _{n \rightarrow \infty}\left\langle f(\bar{x})-\bar{x}, j\left(y_{n}-\bar{x}\right)\right\rangle \leqslant 0$, where $\lim _{t \rightarrow 0} x_{t}=\bar{x}=Q_{\Omega} f(\bar{x})$ and $x_{t}$ solves equation $x_{t}=S J_{r_{n}}^{A}\left(I-r_{n} B\right)\left(t f\left(x_{t}\right)+(1-t) x_{t}\right)$ for all $t \in(0,1)$.

Consider

$$
\begin{aligned}
\left\|W_{n} x_{n}-y_{n}\right\| & \leqslant\left\|S J_{r_{n}}^{A}\left(\left(I-r_{n} B\right)\left[\alpha_{n} f\left(x_{n}\right)+\left(1-\alpha_{n}\right) x_{n}\right]+e_{n}\right)-x_{n}\right\|+\left\|x_{n}-y_{n}\right\| \\
& =\left\|z_{n}-x_{n}\right\|+\left\|x_{n}-y_{n}\right\| .
\end{aligned}
$$

From (3.7) and (3.13), then

$$
\lim _{n \rightarrow \infty}\left\|W_{n} x_{n}-y_{n}\right\|=0
$$

We compute

$$
\begin{aligned}
\left\|x_{t}-y_{n}\right\|^{2}= & \left\|S J_{r_{n}}^{A}\left(I-r_{n} B\right)\left(t f\left(x_{t}\right)+(1-t) x_{t}\right)-y_{n}\right\|^{2} \\
= & \left\langle S J_{r_{n}}^{A}\left(I-r_{n} B\right)\left(t f\left(x_{t}\right)+(1-t) x_{t}\right)-W_{n} x_{n}+W_{n} x_{n}-y_{n}, j\left(x_{t}-y_{n}\right)\right\rangle \\
= & \left\langle S J_{r_{n}}^{A}\left(I-r_{n} B\right)\left(t f\left(x_{t}\right)+(1-t) x_{t}\right)-W_{n} x_{n}, j\left(x_{t}-y_{n}\right)\right\rangle+\left\langle W_{n} x_{n}-y_{n}, j\left(x_{t}-y_{n}\right)\right\rangle \\
= & \left\langle S J_{r_{n}}^{A}\left(I-r_{n} B\right)\left(t f\left(x_{t}\right)+(1-t) x_{t}\right)-S J_{r_{n}}^{A}\left(\left(I-r_{n} B\right) y_{n}+e_{n}\right), j\left(x_{t}-y_{n}\right)\right\rangle \\
& +\left\langle W_{n} x_{n}-y_{n}, j\left(x_{t}-y_{n}\right)\right\rangle \\
\leqslant & \left\langle\left(I-r_{n} B\right)\left(t f\left(x_{t}\right)+(1-t) x_{t}\right)-\left(I-r_{n} B\right) y_{n}-e_{n}, j\left(x_{t}-y_{n}\right)\right\rangle \\
& +\left\|W_{n} x_{n}-y_{n}\right\|\left\|x_{t}-y_{n}\right\| \\
= & \left\langle\left(I-r_{n} B\right)\left(t f\left(x_{t}\right)+(1-t) x_{t}\right)-\left(I-r_{n} B\right) y_{n}, j\left(x_{t}-y_{n}\right)\right\rangle+\left\langle e_{n}, j\left(x_{t}-y_{n}\right)\right\rangle \\
& +\left\|W_{n} x_{n}-y_{n}\right\|\left\|x_{t}-y_{n}\right\| \\
\leqslant & \left\langle\left(t f\left(x_{t}\right)+(1-t) x_{t}\right)-x_{t}+x_{t}-y_{n}, j\left(x_{t}-y_{n}\right)\right\rangle \\
& +\left\|e_{n}\right\|\left\|x_{t}-y_{n}\right\|+\left\|W_{n} x_{n}-y_{n}\right\|\left\|x_{t}-y_{n}\right\|
\end{aligned}
$$




$$
\begin{aligned}
\leqslant & \left\langle t\left(f\left(x_{t}\right)-x_{t}\right), j\left(x_{t}-y_{n}\right)\right\rangle+\left\langle x_{t}-y_{n}, j\left(x_{t}-y_{n}\right)\right\rangle+\left\|e_{n}\right\|\left\|x_{t}-y_{n}\right\| \\
& +\left\|W_{n} x_{n}-y_{n}\right\|\left\|x_{t}-y_{n}\right\| \\
\leqslant & t\left\langle f\left(x_{t}\right)-x_{t}, j\left(x_{t}-y_{n}\right)\right\rangle+\left\|x_{t}-y_{n}\right\|^{2}+\left\|e_{n}\right\|\left\|x_{t}-y_{n}\right\|+\left\|W_{n} x_{n}-y_{n}\right\|\left\|x_{t}-y_{n}\right\| \\
\leqslant & -t\left\langle f\left(x_{t}\right)-x_{t}, j\left(y_{n}-x_{t}\right)\right\rangle+\left\|x_{t}-y_{n}\right\|^{2}+\left\|e_{n}\right\|\left\|x_{t}-y_{n}\right\|+\left\|W_{n} x_{n}-y_{n}\right\|\left\|x_{t}-y_{n}\right\| .
\end{aligned}
$$

It follows that

$$
t\left\langle f\left(x_{t}\right)-x_{t}, j\left(y_{n}-x_{t}\right)\right\rangle \leqslant\left\|e_{n}\right\|\left\|x_{t}-y_{n}\right\|+\left\|W_{n} x_{n}-y_{n}\right\|\left\|x_{t}-y_{n}\right\| .
$$

Then

$$
\left\langle f\left(x_{t}\right)-x_{t}, j\left(y_{n}-x_{t}\right)\right\rangle \leqslant \frac{1}{t}\left\{\left\|e_{n}\right\|\left\|x_{t}-y_{n}\right\|+\left\|W_{n} x_{n}-y_{n}\right\|\left\|x_{t}-y_{n}\right\|\right\} .
$$

By virtue of (3.17) and condition (d), we get that

$$
\limsup _{n \rightarrow \infty}\left\langle f\left(x_{t}\right)-x_{t}, j\left(y_{n}-x_{t}\right)\right\rangle \leqslant 0 .
$$

Since $x_{t} \rightarrow \bar{x}$, as $t \rightarrow 0$ and $j$ is norm-to-weak* uniformly continuous on bounded subset of $E$, we obtain that

$$
\begin{aligned}
& \left|\left\langle f(\bar{x})-\bar{x}, j\left(y_{n}-\bar{x}\right)\right\rangle-\left\langle f\left(x_{t}\right)-x_{t}, j\left(y_{n}-x_{t}\right)\right\rangle\right| \\
& \quad \leqslant\left|\left\langle f(\bar{x})-\bar{x}, j\left(y_{n}-\bar{x}\right)\right\rangle-\left\langle f(\bar{x})-\bar{x}, j\left(y_{n}-x_{t}\right)\right\rangle\right|+\left|\left\langle f(\bar{x})-\bar{x}, j\left(y_{n}-x_{t}\right)\right\rangle-\left\langle f\left(x_{t}\right)-x_{t}, j\left(y_{n}-x_{t}\right)\right\rangle\right| \\
& \quad \leqslant\left|\left\langle f(\bar{x})-\bar{x}, j\left(y_{n}-\bar{x}\right)-j\left(y_{n}-x_{t}\right)\right\rangle\right|+\left|\left\langle f(\bar{x})-\bar{x}-f\left(x_{t}\right)+x_{t}, j\left(y_{n}-x_{t}\right)\right\rangle\right| \\
& \quad \leqslant\|f(\bar{x})-\bar{x}\|\left\|j\left(y_{n}-\bar{x}\right)-j\left(y_{n}-x_{t}\right)\right\|+\left\|f(\bar{x})-\bar{x}-f\left(x_{t}\right)+x_{t}\right\|\left\|y_{n}-x_{t}\right\| \rightarrow 0, \text { as } t \rightarrow 0 .
\end{aligned}
$$

Hence, for any $\epsilon>0$, there exists $\delta>0$ with some $t \in(0, \delta)$ such that

$$
\left\langle f(\bar{x})-\bar{x}, j\left(y_{n}-\bar{x}\right)\right\rangle \leqslant\left\langle f\left(x_{t}\right)-x_{t}, j\left(y_{n}-x_{t}\right)\right\rangle+\epsilon .
$$

Then, we obtain that

$$
\limsup _{n \rightarrow \infty}\left\langle f(\bar{x})-\bar{x}, j\left(y_{n}-\bar{x}\right)\right\rangle \leqslant \limsup _{n \rightarrow \infty}\left\langle f\left(x_{t}\right)-x_{t}, j\left(y_{n}-x_{t}\right)\right\rangle+\epsilon .
$$

Since $\epsilon$ is arbitrary, by (3.18), we obtain that

$$
\limsup _{n \rightarrow \infty}\left\langle f(\bar{x})-\bar{x}, j\left(y_{n}-\bar{x}\right)\right\rangle \leqslant 0 .
$$

Step 6. Next, we will prove that $\left\{x_{n}\right\}$ converges strongly to $\bar{x}=Q_{\Omega} f(\bar{x})$ by using Lemma 2.3 and Lemma 2.9. We note that

$$
\begin{aligned}
\left\|x_{n+1}-\bar{x}\right\|^{2}= & \left\|\beta_{n} x_{n}+\left(1-\beta_{n}\right) S J_{r_{n}}^{A}\left(v_{n}\right)-\bar{x}\right\|^{2} \\
\leqslant & \beta_{n}\left\|x_{n}-\bar{x}\right\|^{2}+\left(1-\beta_{n}\right)\left\|S J_{r_{n}}^{A}\left(v_{n}\right)-\bar{x}\right\|^{2} \\
= & \beta_{n}\left\|x_{n}-\bar{x}\right\|^{2}+\left(1-\beta_{n}\right)\left\|S J_{r_{n}}^{A}\left(v_{n}\right)-S \bar{x}\right\|^{2} \\
\leqslant & \beta_{n}\left\|x_{n}-\bar{x}\right\|^{2}+\left(1-\beta_{n}\right)\left\|J_{r_{n}}^{A}\left(v_{n}\right)-\bar{x}\right\|^{2} \\
= & \beta_{n}\left\|x_{n}-\bar{x}\right\|^{2}+\left(1-\beta_{n}\right)\left\|J_{r_{n}}^{A}\left(v_{n}\right)-J_{r_{n}}^{A}\left(I-r_{n} B\right) \bar{x}\right\|^{2} \\
\leqslant & \beta_{n}\left\|x_{n}-\bar{x}\right\|^{2}+\left(1-\beta_{n}\right)\left\|v_{n}-\left(I-r_{n} B\right) \bar{x}\right\|^{2} \\
= & \beta_{n}\left\|x_{n}-\bar{x}\right\|^{2}+\left(1-\beta_{n}\right)\left\|\left(y_{n}-r_{n} B y_{n}+e_{n}\right)-\left(I-r_{n} B\right) \bar{x}\right\|^{2} \\
= & \beta_{n}\left\|x_{n}-\bar{x}\right\|^{2}+\left(1-\beta_{n}\right)\left\|\left(I-r_{n} B\right) y_{n}-\left(I-r_{n} B\right) \bar{x}+e_{n}\right\|^{2} \\
= & \beta_{n}\left\|x_{n}-\bar{x}\right\|^{2}+\left(1-\beta_{n}\right)\left[\left\|\left(I-r_{n} A\right) y_{n}-\left(I-r_{n} A\right) \bar{x}\right\|^{2}\right. \\
& +2\left\langle e_{n}, j\left(\left(I-r_{n} B\right) y_{n}-\left(I-r_{n} B\right) \bar{x}+e_{n}\right)\right\rangle \\
\leqslant & \beta_{n}\left\|x_{n}-\bar{x}\right\|^{2}+\left(1-\beta_{n}\right)\left[\left\|y_{n}-\bar{x}\right\|^{2}+2\left\|e_{n}\right\|\left\|\left(I-r_{n} B\right) y_{n}-\left(I-r_{n} B\right) \bar{x}+e_{n}\right\|\right] .
\end{aligned}
$$


Consider

$$
\begin{aligned}
\left\|y_{n}-\bar{x}\right\|^{2} & =\left\langle\alpha_{n} f\left(x_{n}\right)+\left(1-\alpha_{n}\right) x_{n}-\bar{x}, j\left(y_{n}-\bar{x}\right)\right\rangle \\
& =\left\langle\alpha_{n}\left(f\left(x_{n}\right)-\bar{x}\right)+\left(1-\alpha_{n}\right)\left(x_{n}-\bar{x}\right), j\left(y_{n}-\bar{x}\right)\right\rangle \\
& =\left\langle\alpha_{n}\left(f\left(x_{n}\right)-f(\bar{x})\right)+\alpha_{n}(f(\bar{x})-\bar{x})+\left(1-\alpha_{n}\right)\left(x_{n}-\bar{x}\right), j\left(y_{n}-\bar{x}\right)\right\rangle \\
& =\left\langle\alpha_{n}\left(f\left(x_{n}\right)-f(\bar{x})\right)+\left(1-\alpha_{n}\right)\left(x_{n}-\bar{x}\right), j\left(y_{n}-\bar{x}\right)\right\rangle+\left\langle\alpha_{n}(f(\bar{x})-\bar{x}), j\left(y_{n}-\bar{x}\right)\right\rangle \\
& \leqslant\left\|\alpha_{n}\left(f\left(x_{n}\right)-f(\bar{x})\right)+\left(1-\alpha_{n}\right)\left(x_{n}-\bar{x}\right)\right\|\left\|y_{n}-\bar{x}\right\|+\alpha_{n}\left\langle f(\bar{x})-\bar{x}, j\left(y_{n}-\bar{x}\right)\right\rangle \\
& \leqslant\left[\alpha_{n} k\left\|x_{n}-\bar{x}\right\|+\left(1-\alpha_{n}\right)\left\|x_{n}-\bar{x}\right\|\right]\left\|y_{n}-\bar{x}\right\|+\alpha_{n}\left\langle f(\bar{x})-\bar{x}, j\left(y_{n}-\bar{x}\right)\right\rangle \\
& =\left[1-\alpha_{n}(1-k)\right]\left\|x_{n}-\bar{x}\right\|\left\|y_{n}-\bar{x}\right\|+\alpha_{n}\left\langle f(\bar{x})-\bar{x}, j\left(y_{n}-\bar{x}\right)\right\rangle \\
& =\left(1-\alpha_{n}(1-k)\right) \frac{\left\|x_{n}-\bar{x}\right\|^{2}+\left\|y_{n}-\bar{x}\right\|^{2}}{2}+\alpha_{n}\left\langle f(\bar{x})-\bar{x}, j\left(y_{n}-\bar{x}\right)\right\rangle \\
& =\frac{1-\alpha_{n}(1-k)}{2}\left(\left\|x_{n}-\bar{x}\right\|^{2}+\left\|y_{n}-\bar{x}\right\|^{2}\right)+\alpha_{n}\left\langle f(\bar{x})-\bar{x}, j\left(y_{n}-\bar{x}\right)\right\rangle .
\end{aligned}
$$

It follows that

$$
\begin{aligned}
2\left\|y_{n}-\bar{x}\right\|^{2} & \leqslant\left(1-\alpha_{n}(1-k)\right)\left\|x_{n}-\bar{x}\right\|^{2}+\left(1-\alpha_{n}(1-k)\right)\left\|y_{n}-\bar{x}\right\|^{2}+2 \alpha_{n}\left\langle f(\bar{x})-\bar{x}, j\left(y_{n}-\bar{x}\right)\right\rangle \\
& \leqslant\left(1-\alpha_{n}(1-k)\right)\left\|x_{n}-\bar{x}\right\|^{2}+\left\|y_{n}-\bar{x}\right\|^{2}+2 \alpha_{n}\left\langle f(\bar{x})-\bar{x}, j\left(y_{n}-\bar{x}\right)\right\rangle .
\end{aligned}
$$

Therefore, we obtain that

$$
\left\|y_{n}-\bar{x}\right\|^{2} \leqslant\left(1-\alpha_{n}(1-k)\right)\left\|x_{n}-\bar{x}\right\|^{2}+2 \alpha_{n}\left\langle f(\bar{x})-\bar{x}, j\left(y_{n}-\bar{x}\right)\right\rangle .
$$

By (3.19) and (3.20), we conclude that

$$
\begin{aligned}
\left\|x_{n+1}-\bar{x}\right\|^{2} \leqslant & \beta_{n}\left\|x_{n}-\bar{x}\right\|^{2}+\left(1-\beta_{n}\right)\left[\left(1-\alpha_{n}(1-k)\right)\left\|x_{n}-\bar{x}\right\|^{2}+2 \alpha_{n}\left\langle f(\bar{x})-\bar{x}, j\left(y_{n}-\bar{x}\right)\right\rangle\right] \\
& +\left(1-\beta_{n}\right) 2\left\|e_{n}\right\|\left\|\left(I-r_{n} B\right) y_{n}-\left(I-r_{n} B\right) \bar{x}+e_{n}\right\| \\
= & \left(1-\alpha_{n}(1-k)\left(1-\beta_{n}\right)\right)\left\|x_{n}-\bar{x}\right\|^{2}+2 \alpha_{n}\left(1-\beta_{n}\right)\left\langle f(\bar{x})-\bar{x}, j\left(y_{n}-\bar{x}\right)\right\rangle \\
& +2\left(1-\beta_{n}\right)\left\|e_{n}\right\|\left\|\left(I-r_{n} B\right) y_{n}-\left(I-r_{n} B\right) \bar{x}+e_{n}\right\| \\
= & \left(1-\lambda_{n}\right)\left\|x_{n}-\bar{x}\right\|^{2}+\frac{2 \lambda_{n}}{(1-k)}\left\langle f(\bar{x})-\bar{x}, j\left(y_{n}-\bar{x}\right)\right\rangle+c_{n},
\end{aligned}
$$

where $c_{n}:=2\left(1-\beta_{n}\right)\left\|e_{n}\right\|\left\|\left(I-r_{n} B\right) y_{n}-\left(I-r_{n} B\right) \bar{x}+e_{n}\right\|$ and $\lambda_{n}=\alpha_{n}(1-k)\left(1-\beta_{n}\right)$.

Setting $b_{n}=\frac{2}{(1-k)}\left\langle f(\bar{x})-\bar{x}, j\left(y_{n}-\bar{x}\right)\right\rangle$, since $\limsup _{n \rightarrow \infty}\left\langle f(\bar{x})-\bar{x}, j\left(y_{n}-\bar{x}\right)\right\rangle \leqslant 0$ then we see that $\limsup _{n \rightarrow \infty} b_{n} \leqslant 0$, and also that $\sum_{n=0}^{\infty} c_{n}<\infty$.

By Lemma 2.8 and conditions (a), (b), and (d), we conclude that $\left\|x_{n}-\bar{x}\right\|^{2} \rightarrow 0$, as $n \rightarrow \infty$. This implies

$$
\lim _{n \rightarrow \infty}\left\|x_{n}-\bar{x}\right\|=0,
$$

i.e., $x_{n}$ converges strongly to $\bar{x}$.

Next, we will utilize Theorem 3.2 to study some strong convergence theorem in $\mathrm{L}_{p}$ with $2 \leqslant p<\infty$. Since $L_{p}$, where $p \geqslant 2$ are uniformly convex and 2-uniformly smooth Banach space with $K=p-1$, then we consider $\mathrm{E}=\mathrm{L}_{\mathrm{p}}$ and we derive the following theorem.

Theorem 3.3. Let $\mathrm{C}$ be a nonempty closed convex subset of an $\mathrm{L}_{\mathrm{p}}$ for $2 \leqslant \mathrm{p}<\infty$. Let $\mathrm{A}, \mathrm{B}, \mathrm{S}, \mathrm{f}, \mathrm{J}_{\mathrm{r}_{\mathrm{n}}}^{\mathrm{A}}$ be the same as in Theorem 3.2. Let $\left\{\alpha_{n}\right\},\left\{\beta_{n}\right\}$ be real number sequences in $(0,1),\left\{r_{n}\right\}$ is a real number sequence in $\left(0, \frac{\alpha}{(p-1)^{2}}\right)$ and $\left\{e_{n}\right\}$ is a sequence in $\mathrm{E}$. Assume that the control sequences satisfy the following $(a),(b),(d)$ in Theorem 3.2 , and condition (c) $\lim _{n \rightarrow \infty} r_{n}=r$, and $r \in\left(0, \frac{\alpha}{(p-1)^{2}}\right)$. Then the sequence $\left\{x_{n}\right\}$ defined by (3.1) converges strongly to a point $\bar{x} \in \operatorname{Fix}(S) \cap(A+B)^{-1}(0)$. 
Consider a mapping $\mathrm{S} \equiv \mathrm{I}$ in Theorem 3.2, we can obtain the following corollary directly.

Corollary 3.4. Let $\mathrm{E}$ be a uniformly convex and 2-uniformly smooth Banach space with weakly sequentially continuous duality mapping. Let $\mathrm{C}$ be a nonempty closed convex subset of $\mathrm{E}$. Let $\mathrm{A}: \mathrm{D}(\mathrm{A}) \subseteq \mathrm{E} \rightarrow 2^{\mathrm{E}}$ be an m-accretive operator such that the domain of $\mathrm{A}$ is included in $\mathrm{C}$ and $\mathrm{B}: \mathrm{C} \rightarrow \mathrm{X}$ be an $\alpha$-inverse strongly accretive operator. Let $\mathrm{f}: \mathrm{C} \rightarrow \mathrm{C}$ be a contraction mapping with the constant $\mathrm{k} \in(0,1)$. Let $\mathrm{J}_{\mathrm{r}_{n}}^{\mathrm{A}}=\left(\mathrm{I}+\mathrm{r}_{\mathrm{n}} \mathrm{A}\right)^{-1}$ be a resolvent of $\mathrm{A}$ for $r_{\mathrm{n}}>0$ such that $(\mathrm{A}+\mathrm{B})^{-1}(0) \neq \emptyset$.

For given $\mathrm{x}_{0} \in \mathrm{C}$, let $\mathrm{x}_{\mathrm{n}}$ be a sequence in the following process:

$$
\left\{\begin{array}{l}
y_{n}=\alpha_{n} f\left(x_{n}\right)+\left(1-\alpha_{n}\right) x_{n}, \\
x_{n+1}=\beta_{n} x_{n}+\left(1-\beta_{n}\right) J_{r_{n}}^{A}\left(y_{n}-r_{n} B y_{n}+e_{n}\right), \quad \forall n \geqslant 0,
\end{array}\right.
$$

where $\left\{\alpha_{n}\right\},\left\{\beta_{n}\right\}$ are real number sequences in $(0,1),\left\{r_{n}\right\}$ is a real number sequence in $\left(0, \frac{\alpha}{K^{2}}\right), K>0$ is the 2uniformly smooth constant of $\mathrm{E}$ and $\left\{\mathrm{e}_{\mathrm{n}}\right\}$ is a sequence in $\mathrm{E}$. Assume that the control sequences satisfy the following conditions:

(a) $\lim _{n \rightarrow \infty} \alpha_{n}=0, \sum_{n=1}^{\infty} \alpha_{n}=\infty$;

(b) $0<\liminf _{n \rightarrow \infty} \beta_{n} \leqslant \limsup _{n \rightarrow \infty} \beta_{n}<1$;

(c) $\lim _{n \rightarrow \infty} r_{n}=r$, and $r \in\left(0, \frac{\alpha}{\mathrm{K}^{2}}\right)$;

(d) $\sum_{n=0}^{\infty}\left\|e_{n}\right\|<\infty$.

Then, the sequence $\left\{x_{n}\right\}$ converges strongly to a point $\bar{x} \in(A+B)^{-1}(0)$.

Consider a mapping $S \equiv I$ and $f\left(x_{n}\right) \equiv u$ for all $n \in \mathbb{N}$ in Theorem 3.2, we obtain the following corollary directly.

Corollary 3.5. Let $\mathrm{E}$ be a uniformly convex and 2-uniformly smooth Banach space with weakly sequentially continuous duality mapping. Let $\mathrm{C}$ be a nonempty closed convex subset of $\mathrm{E}$. Let $\mathrm{A}: \mathrm{D}(\mathrm{A}) \subseteq \mathrm{E} \rightarrow 2^{\mathrm{E}}$ be an m-accretive operator such that the domain of $\mathrm{A}$ is included in $\mathrm{C}$ and let $\mathrm{B}: \mathrm{C} \rightarrow \mathrm{X}$ be an $\alpha$-inverse strongly accretive operator. Let $\mathrm{J}_{r_{n}}^{B}=\left(I+r_{n} B\right)^{-1}$ be a resolvent of $B$ for $r_{n}>0$ such that $(A+B)^{-1}(0) \neq \emptyset$.

For given $\mathrm{x}_{0} \in \mathrm{C}$, let $\mathrm{x}_{\mathrm{n}}$ be a sequence in the following process:

$$
\left\{\begin{array}{l}
y_{n}=\alpha_{n} u+\left(1-\alpha_{n}\right) x_{n}, \\
x_{n+1}=\beta_{n} x_{n}+\left(1-\beta_{n}\right) J_{r_{n}}^{A}\left(y_{n}-r_{n} B y_{n}+e_{n}\right), \quad \forall n \geqslant 0,
\end{array}\right.
$$

where $\left\{\alpha_{n}\right\},\left\{\beta_{n}\right\}$ are real number sequences in $(0,1),\left\{r_{n}\right\}$ is a real number sequence in $\left(0, \frac{\alpha}{K^{2}}\right), K>0$ is the 2uniformly smooth constant of $\mathrm{E}$ and $\left\{\mathrm{e}_{\mathrm{n}}\right\}$ is a sequence in $\mathrm{E}$. Assume that the control sequence satisfies the following conditions:

(a) $0<\liminf _{n \rightarrow \infty} \beta_{n} \leqslant \limsup _{n \rightarrow \infty} \beta_{n}<1$;

(b) $\lim _{n \rightarrow \infty} r_{n}=r$, and $r \in\left(0, \frac{\alpha}{K^{2}}\right)$;

(c) $\sum_{n=0}^{\infty}\left\|e_{n}\right\|<\infty$.

Then, the sequence $\left\{x_{n}\right\}$ converges strongly to a point $\bar{x} \in(A+B)^{-1}(0)$.

Setting $J_{r_{n}}^{A} \equiv I, B \equiv 0, f\left(x_{n}\right) \equiv u$ for all $n \in \mathbb{N}$ and $e_{n} \equiv 0$, then we have the following corollary of the modified Mann-Halpern iteration.

Corollary 3.6. Let $\mathrm{E}$ be a uniformly convex and 2-uniformly smooth Banach space and let $\mathrm{C}$ be a nonempty closed convex subset of $\mathrm{E}$. Let $\mathrm{S}: \mathrm{C} \rightarrow \mathrm{C}$ be a nonexpansive mapping such that $\operatorname{Fix}(\mathrm{S}) \neq \emptyset$. For given $\mathrm{x}_{0}, \mathrm{u} \in \mathrm{C}$, let $\mathrm{x}_{\mathrm{n}}$ be a sequence in the following process:

$$
\left\{\begin{array}{l}
y_{n}=\alpha_{n} u+\left(1-\alpha_{n}\right) x_{n}, \\
x_{n+1}=\beta_{n} x_{n}+\left(1-\beta_{n}\right) S y_{n}, \quad \forall n \geqslant 0,
\end{array}\right.
$$

where $\left\{\alpha_{n}\right\},\left\{\beta_{n}\right\}$ are real number sequences in $(0,1)$. Assume that the control sequence satisfies the following conditions: 
(a) $\lim _{n \rightarrow \infty} \alpha_{n}=0$, and $\sum_{n=1}^{\infty} \alpha_{n}=\infty$;

(b) $0<\liminf _{n \rightarrow \infty} \beta_{n} \leqslant \limsup _{n \rightarrow \infty} \beta_{n}<1$.

Then, the sequence $\left\{x_{n}\right\}$ converges strongly to a point $\bar{x} \in \operatorname{Fix}(S)$.

\section{Some applications}

In this section, we give two applications of our main results in the framework of Hilbert spaces. Now, we consider Theorem 3.2, in the framework of Hilbert spaces, it is known that $K=\frac{\sqrt{2}}{2}$. Let $\mathrm{H}$ be a Hilbert space and let $\mathrm{C}$ be a nonempty closed convex subset of $\mathrm{H}$.

Theorem 4.1 ([5, Corollary 2.2]). Let $\mathrm{A}: \mathrm{C} \rightarrow 2^{\mathrm{H}}$ be a maximal monotone operator such that the domain of $\mathrm{B}$ which is included in $\mathrm{C}$ and $\mathrm{B}: \mathrm{C} \rightarrow \mathrm{H}$ be an $\alpha$-inverse strongly monotone operator. Let $\mathrm{S}: \mathrm{C} \rightarrow \mathrm{C}$ be a nonexpansive mapping and let $\mathrm{f}: \mathrm{C} \rightarrow \mathrm{C}$ be a contraction mapping with the constant $\mathrm{k} \in(0,1)$. Let $\mathrm{J}_{\mathrm{r}_{\mathrm{n}}}^{\mathrm{A}}=\left(\mathrm{I}+\mathrm{r}_{\mathrm{n}} \mathrm{A}\right)^{-1}$ be a resolvent of $A$ for $r_{n}>0$ such that $\operatorname{Fix}(S) \cap(A+B)^{-1}(0) \neq \emptyset$.

For given $x_{0} \in C$, let $\left\{x_{n}\right\}$ be a sequence defined by following:

$$
\left\{\begin{array}{l}
y_{n}=\alpha_{n} f\left(x_{n}\right)+\left(1-\alpha_{n}\right) x_{n}, \\
x_{n+1}=\beta_{n} x_{n}+\left(1-\beta_{n}\right) S J_{r_{n}}^{A}\left(y_{n}-r_{n} B y_{n}+e_{n}\right), \forall n \geqslant 0,
\end{array}\right.
$$

where $\left\{\alpha_{n}\right\},\left\{\beta_{n}\right\}$ are real number sequences in $(0,1),\left\{r_{n}\right\}$ is a real number sequence in $(0,2 \alpha)$ and $\left\{e_{n}\right\}$ is a sequence in $\mathrm{H}$. Assume that the control sequences satisfy the following conditions:

(a) $\lim _{n \rightarrow \infty} \alpha_{n}=0$, and $\sum_{n=1}^{\infty} \alpha_{n}=\infty$;

(b) $0<\liminf _{n \rightarrow \infty} \beta_{n} \leqslant \limsup _{n \rightarrow \infty} \beta_{n}<1$;

(c) $\lim _{n \rightarrow \infty} r_{n}=r$, and $r \in\left(0, \frac{\alpha}{\mathrm{K}^{2}}\right)$;

(d) $\sum_{n=0}^{\infty}\left\|e_{n}\right\|<\infty$.

Then, the sequence $\left\{x_{n}\right\}$ converges strongly to a point $\bar{x} \in \operatorname{Fix}(S) \cap(A+B)^{-1}(0)$. Next, we will give some related results.

\subsection{Application to projection for variational inequality}

Let $\mathrm{C}$ be a nonempty, closed, and convex subset of a Hilbert space $\mathrm{H}$. The metric projection of a point $x \in \mathrm{H}$ onto $\mathrm{C}$, denoted by $\mathrm{P}_{\mathrm{C}}(\mathrm{x})$, is defined as the unique solution of the problem

$$
\left\|x-P_{C} x\right\| \leqslant\|x-y\|, \quad \forall y \in C, \quad \forall x \in H .
$$

For each $x \in H$ and $z \in C$, the metric projection $\mathrm{P}_{\mathrm{C}}$ satisfies

$$
z=\mathrm{P}_{\mathrm{C}}(x) \Longleftrightarrow\langle y-z, x-z\rangle \leqslant 0, \forall y \in C
$$

Note that the metric projection is nonexpansive mapping.

Let $g: H \rightarrow(-\infty, \infty]$ be a proper convex lower semicontinuous function. Then the subdifferential $\partial g$ of $\mathrm{g}$ is defined as follows,

$$
\partial g(x)=\{z \in H: g(y)-g(x) \geqslant\langle y-x, z\rangle, \forall y \in H\}
$$

for all $x \in H$. If $g(x)=\infty$, then $\partial g(x) \neq \emptyset$, Takahashi [21] claimed that $\partial g$ is $m$-accretive operator. Since we know that, an m-accretive operator is maximal monotone operator in a Hilbert space, then we claim that $\partial g$ is maximal monotone operator. Then we define the set of minimizers of $g$ as follows:

$$
\operatorname{argmin}_{y \in H} g(y)=\left\{z \in H: g(z)=\min _{y \in H} g(y)\right\}
$$


It is easy to verify that $0 \in \partial g(x)$ if and only if $g(z)=\min _{y \in H} g(y)$. Let $i_{C}$ be the indicator function of $C$ by

$$
i_{C}(x)= \begin{cases}0, & x \in C, \\ +\infty, & x \notin C .\end{cases}
$$

Then $i_{C}$ is a proper lower semicontinuous convex function on $H$. So, we see that the subdifferential $\partial i_{C}$ of $i_{C}$ is maximal monotone operator; see [21]. The resolvent $J_{r}$ of $\partial i_{C}$ for $r>0$, that is $J_{r} x=\left(I+r \partial i_{C}\right)^{-1} x$ for all $x \in H$. Next, we recall that set $N_{C}(u)$ is called the normal cone of $C$ at $u$ defined by

$$
\mathrm{N}_{\mathrm{C}}(\mathrm{u})=\{z \in \mathrm{H}:\langle z, y-u\rangle \leqslant 0, \forall y \in C\} .
$$

Since $N_{C}(u)=\partial i_{C}(u)$. In fact, we have that for any $x \in H$ and $u \in C$,

$$
\begin{aligned}
u=\mathrm{J}_{r} x=\left(I+r \partial i_{C}\right)^{-1} x & \Longleftrightarrow x \in u+r \partial i_{C} u \\
& \Longleftrightarrow x \in u+r N_{C}(u) \\
& \Longleftrightarrow x-u \in r N_{C}(u) \\
& \Longleftrightarrow \frac{1}{r}\langle x-u, y-u\rangle \leqslant 0, \quad \forall y \in C \\
& \Longleftrightarrow\langle x-u, y-u\rangle \leqslant 0, \quad \forall y \in C \\
& \Longleftrightarrow u=P_{C} x .
\end{aligned}
$$

Then $u=\left(I+r \partial i_{C}\right)^{-1} \chi \Longleftrightarrow u=P_{C} x$ for all $x \in H, u \in C$.

Now, we consider the following variational inequality problem (VIP) for $B$ is to find $x \in C$ such that

$$
\langle\mathrm{B} x, y-x\rangle \geqslant 0, \forall y \in C \text {. }
$$

The set of solutions of (4.2) is denoted by $\mathrm{VI}(\mathrm{C}, \mathrm{B})$.

$$
\mathrm{VI}(\mathrm{C}, \mathrm{B})=\{\mathrm{x} \in \mathrm{C}:\langle\mathrm{B} x, \mathrm{y}-\mathrm{x}\rangle \geqslant 0, \quad \forall \mathrm{y} \in \mathrm{C}\} .
$$

Theorem 4.2. Let $\mathrm{B}: \mathrm{C} \rightarrow \mathrm{H}$ be an $\alpha$-inverse strongly monotone mapping. Let $\mathrm{S}: \mathrm{C} \rightarrow \mathrm{C}$ be a nonexpansive mapping and let $\mathrm{f}: \mathrm{C} \rightarrow \mathrm{C}$ be a contraction mapping with the constant $\mathrm{k} \in(0,1)$. Assume that $\operatorname{Fix}(\mathrm{S}) \cap \mathrm{VI}(\mathrm{C}, \mathrm{B}) \neq$ $\emptyset$. For given $\mathrm{x}_{0} \in \mathrm{C}$, let $\left\{\mathrm{x}_{\mathrm{n}}\right\}$ be a sequence defined by following:

$$
\left\{\begin{array}{l}
y_{n}=\alpha_{n} f\left(x_{n}\right)+\left(1-\alpha_{n}\right) x_{n}, \\
x_{n+1}=\beta_{n} x_{n}+\left(1-\beta_{n}\right) S P_{C}\left(y_{n}-r_{n} B y_{n}+e_{n}\right), \quad \forall n \geqslant 0,
\end{array}\right.
$$

where $\left\{\alpha_{n}\right\},\left\{\beta_{n}\right\}$ are real number sequences in $(0,1),\left\{r_{n}\right\}$ is a real number sequence in $(0,2 \alpha)$ and $\left\{e_{n}\right\}$ is a sequence in $\mathrm{H}$. Assume that the control sequences satisfy the following conditions:

(a) $\lim _{n \rightarrow \infty} \alpha_{n}=0$, and $\sum_{n=1}^{\infty} \alpha_{n}=\infty$;

(b) $0<\liminf _{n \rightarrow \infty} \beta_{n} \leqslant \limsup _{n \rightarrow \infty} \beta_{n}<1$;

(c) $\lim _{n \rightarrow \infty} r_{n}=r$, and $r \in(0,2 \alpha)$;

(d) $\sum_{n=0}^{\infty}\left\|e_{n}\right\|<\infty$.

Then, the sequence $\left\{x_{n}\right\}$ converges strongly to a point $\bar{x} \in \operatorname{Fix}(S) \cap \operatorname{VI}(C, A)$, where $\bar{x}=P_{\operatorname{Fix}(S) \cap V I(C, B)} f(\bar{x})$.

Proof. By Lemma 2.4 we know that $\operatorname{Fix}\left(J_{r}^{A}(I-r B)\right)=(A+B)^{-1}(0)$. Put $A=\partial i_{C}$, and we show that $\mathrm{VI}(\mathrm{C}, \mathrm{B})=\left(\partial i_{\mathrm{C}}+\mathrm{B}\right)^{-1}(0)$. Note that

$$
\begin{aligned}
x \in\left(\partial i_{C}+B\right)^{-1}(0) & \Longleftrightarrow 0 \in \partial i_{C} x+B x \\
& \Longleftrightarrow 0 \in N_{C} x+B x \\
& \Longleftrightarrow-B x \in N_{C} x \\
& \Longleftrightarrow\langle-B x, y-x\rangle \leqslant 0 \\
& \Longleftrightarrow\langle B x, y-x\rangle \geqslant 0 \\
& \Longleftrightarrow x \in \operatorname{VI}(C, B) .
\end{aligned}
$$

From (4.1), therefore, we can conclude the desired conclusion immediately. 


\subsection{Application for equilibrium problems}

Let $\mathrm{F}$ be a bifunction of $\mathrm{C} \times \mathrm{C}$ into $\mathbb{R}$, where $\mathbb{R}$ is the set of real numbers. The equilibrium problem is to find $x \in \mathrm{C}$ such that

$$
F(x, y) \geqslant 0, \forall y \in C
$$

The set of solutions of (4.3) is denoted by EP(F).

For solving the equilibrium problem, we assume that the bifunction $F$ satisfies the following conditions:

$\left(\mathrm{A}_{1}\right) \mathrm{F}(\mathrm{x}, \mathrm{x})=0$ for all $x \in \mathrm{C}$;

$\left(A_{2}\right) F$ is monotone, i.e., $F(x, y)+F(y, x) \leqslant 0$ for any $x, y \in C$;

$\left(A_{3}\right)$ for each $x, y, z \in C$, $\lim \sup _{t \rightarrow 0^{+}} F(t z+(1-t) x, y) \leqslant F(x, y)$;

$\left(\mathrm{A}_{4}\right)$ for each $x \in C, y \mapsto F(x, y)$ is convex and lower semicontinuous.

Lemma 4.3 ([3]). Let $\mathrm{C}$ be a nonempty closed and convex subset of a real Hilbert space $\mathrm{H}$ and let $\mathrm{F}$ be a bifunction of $\mathrm{C} \times \mathrm{C}$ into $\mathbb{R}$ satisfying $\left(\mathrm{A}_{1}\right)-\left(\mathrm{A}_{4}\right)$. Let $\mathrm{r}>0$ and $z \in \mathrm{H}$. Then, there exists $\mathrm{x} \in \mathrm{C}$ such that

$$
\mathrm{F}(x, y)+\frac{1}{r}\langle y-x, x-z\rangle \geqslant 0, \forall y \in C .
$$

Lemma 4.4 ([6]). Let $\mathrm{C}$ be a nonempty closed and convex subset of a real Hilbert space $\mathrm{H}$ and let $\mathrm{F}: \mathrm{C} \times \mathrm{C} \rightarrow \mathbb{R}$ satisfy $\left(\mathrm{A}_{1}\right)-\left(\mathrm{A}_{4}\right)$. For $\mathrm{r}>0$ and $z \in \mathrm{H}$, define a mapping $\mathrm{T}_{\mathrm{r}}: \mathrm{H} \rightarrow \mathrm{C}$ as follows:

$$
\mathrm{T}_{\mathrm{r}}(z)=\left\{x \in \mathrm{C}: \mathrm{F}(\mathrm{x}, \mathrm{y})+\frac{1}{\mathrm{r}}\langle\mathrm{y}-\mathrm{x}, \mathrm{x}-z\rangle \geqslant 0, \forall \mathrm{y} \in \mathrm{C}\right\}, \forall z \in \mathrm{H} .
$$

Then, the following hold:

(1) $\mathrm{T}_{\mathrm{r}}$ is single-valued;

(2) $\mathrm{T}_{\mathrm{r}}$ is firmly nonexpansive, i.e., for any $\mathrm{x}, \mathrm{y} \in \mathrm{H}$,

$$
\left\|T_{r} x-T_{r} y\right\|^{2} \leqslant\left\langle T_{r}(x)-T_{r}(y), x-y\right\rangle ;
$$

(3) $\operatorname{Fix}\left(T_{r}\right)=\operatorname{EP}(F)$;

(4) $\mathrm{EP}(\mathrm{F})$ is closed and convex.

Lemma 4.5 ([22]). Let $\mathrm{C}$ be a nonempty closed and convex subset of a real Hilbert space $\mathrm{H}$ and let $\mathrm{F}: \mathrm{C} \times \mathrm{C} \rightarrow \mathbb{R}$ satisfy $\left(\mathrm{A}_{1}\right)-\left(\mathrm{A}_{4}\right)$ and $\mathrm{A}_{\mathrm{F}}$ be a multi-valued mapping of $\mathrm{H}$ into itself defined by

$$
A_{F} x= \begin{cases}\{z \in H: F(x, y) \geqslant\langle y-x, z\rangle, \forall y \in C\}, & x \in C, \\ \emptyset, & x \notin C .\end{cases}
$$

Then $\mathrm{EP}(\mathrm{F})=\mathrm{A}_{\mathrm{F}}^{-1}(0)$ and $\mathrm{A}_{\mathrm{F}} \mathrm{x}$ is a maximal monotone operator with the domain $\mathrm{D}\left(\mathrm{A}_{\mathrm{F}}\right) \subset \mathrm{C}$. Furthermore, the resolvent $T_{r}$ of $F$ coincides with the resolvent of $A_{F}$, i.e.,

$$
\mathrm{T}_{\mathrm{r}} x=\left(\mathrm{I}+\mathrm{rA} \mathrm{F}_{\mathrm{F}}\right)^{-1}(x), \forall x \in \mathrm{H}, \mathrm{r}>0,
$$

where $\mathrm{T}_{\mathrm{r}}$ is defined as in (4.4).

We recall that $T_{r}$ is the resolvent of $A_{F}$ for $r>0$. Since $A=A_{F}$, we will show that $J_{r} x=T_{r} x$. Indeed, for $x \in H$, we have

$$
\begin{aligned}
z \in \mathrm{J}_{\mathrm{r}} x=\left(\mathrm{I}+\mathrm{r} A_{\mathrm{F}}\right)^{-1}(x) & \Longleftrightarrow x \in\left(\mathrm{I}+\mathrm{r} A_{\mathrm{F}}\right) z \\
& \Longleftrightarrow x \in z+r A_{\mathrm{F}} z \\
& \Longleftrightarrow x-z \in \mathrm{rA} A_{\mathrm{F}} z
\end{aligned}
$$




$$
\begin{aligned}
& \Longleftrightarrow \frac{1}{r}(x-z) \in A_{F} z \\
& \Longleftrightarrow F(z, y) \geqslant\left\langle y-z, \frac{1}{r}(x-z)\right\rangle \\
& \Longleftrightarrow F(z, y) \geqslant\left\langle y-z, \frac{-1}{r}(z-x)\right\rangle \\
& \Longleftrightarrow F(z, y) \geqslant \frac{-1}{r}\langle y-z, z-x\rangle \\
& \Longleftrightarrow F(z, y)+\frac{1}{r}\langle y-z, z-x\rangle \geqslant 0, \forall y \in C \\
& \Longleftrightarrow z \in T_{r} x .
\end{aligned}
$$

Using Lemmas 4.3, 4.4, 4.5 and Theorem 4.1, we also obtain the following result.

Theorem 4.6. Let $\mathrm{F}: \mathrm{C} \times \mathrm{C} \rightarrow \mathbb{R}$ which satisfies $\left(\mathrm{A}_{1}\right)-\left(\mathrm{A}_{4}\right)$. Let $\mathrm{S}: \mathrm{C} \rightarrow \mathrm{C}$ be a nonexpansive mapping and let $\mathrm{f}: \mathrm{C} \rightarrow \mathrm{C}$ be a contraction mapping with the constant $\mathrm{k} \in(0,1)$. Assume that $\operatorname{Fix}(\mathrm{S}) \cap \mathrm{EP}(\mathrm{F}) \neq \emptyset$. For given $\mathrm{x}_{0} \in \mathrm{C}$, let $\left\{\mathrm{x}_{\mathrm{n}}\right\}$ be a sequence defined by following:

$$
\left\{\begin{array}{l}
y_{n}=\alpha_{n} f\left(x_{n}\right)+\left(1-\alpha_{n}\right) x_{n}, \\
x_{n+1}=\beta_{n} x_{n}+\left(1-\beta_{n}\right) S T_{r_{n}}\left(y_{n}+e_{n}\right), \quad \forall n \geqslant 0,
\end{array}\right.
$$

where $\left\{\alpha_{n}\right\},\left\{\beta_{n}\right\}$ are real number sequences in $(0,1),\left\{r_{n}\right\}$ is a real number sequence in $(0,2 \alpha)$ and $\left\{e_{n}\right\}$ is a sequence in $\mathrm{H}$. Assume that the control sequences satisfy the following conditions:

(a) $\lim _{n \rightarrow \infty} \alpha_{n}=0$, and $\sum_{n=1}^{\infty} \alpha_{n}=\infty$;

(b) $0<\liminf _{n \rightarrow \infty} \beta_{n} \leqslant \limsup _{n \rightarrow \infty} \beta_{n}<1$;

(c) $\lim _{n \rightarrow \infty} r_{n}=r$, and $r \in(0,2 \alpha)$;

(d) $\sum_{n=0}^{\infty}\left\|e_{n}\right\|<\infty$.

Then, the sequence $\left\{x_{n}\right\}$ converges strongly to a point $\bar{x} \in \operatorname{Fix}(S) \cap \operatorname{EP}(F)$, where $\bar{x}=P_{\operatorname{Fix}(S) \cap E P(F)} f(\bar{x})$.

Proof. Put $A \equiv A_{F}$ and $B \equiv 0$ in $(A+B)^{-1}(0)$ from Theorem 4.1. Furthermore, for bifunction $F: C \times C \rightarrow$ $\mathbb{R}$, we define $A_{F} x$ as in Lemma 4.5, we have $E P(F)=A_{F}^{-1}(0)$ and let $T_{r_{n}}$ be the resolvent of $A_{F}$ for $r_{n}>0$. Therefore, we can conclude the desired conclusion immediately.

\section{Numerical Example}

In this section, we demonstrate the performance and convergence of Theorem 3.2 with the following example.

Example 5.1. Let $E=\mathbb{R}$ and $C=[-1,000,1,000]$. Define the following mappings

$$
f(x):=0.5 x, \quad A(x):=2 x+1, \quad B(x):=\frac{2}{3}(x-1), \quad \text { and } \quad S(x):=\frac{1}{2} x-\frac{1}{16} x .
$$

We see that the proposed mappings satisfy the assumptions in Theorem 3.2. It is easily seen that for $r>0, J_{r}^{A}(I-r B)(x)=\frac{3 x-2 r x-r}{3+6 r}$. Furthermore, we have a point $-\frac{1}{8}$ which is in the fixed point sets of $S$ and $J_{r}^{A}(I-r B)$, that is $-\frac{1}{8} \in \operatorname{Fix}(S) \cap(A+B)^{-1}(0)$ and can be seen in Figure 1.

In this example, we set the parameters on algorithm (3.1) by $\alpha_{n}=\frac{1}{n+1}, \beta_{n}=\frac{2 n}{3 n+1}, r_{n}=\frac{n+1}{2 n}$ and $e_{n}=0$ for all $n \geqslant 0$. So, $\left\{\alpha_{n}\right\},\left\{\beta_{n}\right\},\left\{r_{n}\right\}$, and $\left\{e_{n}\right\}$ are real number sequences that satisfy all of the conditions (a)-(d) in Theorem 3.2.

We tested the algorithm (3.1) for this example starting three initial points are random and the computation results are reported in Figure 2. The computations associated with example were performed using MATLAB software. 


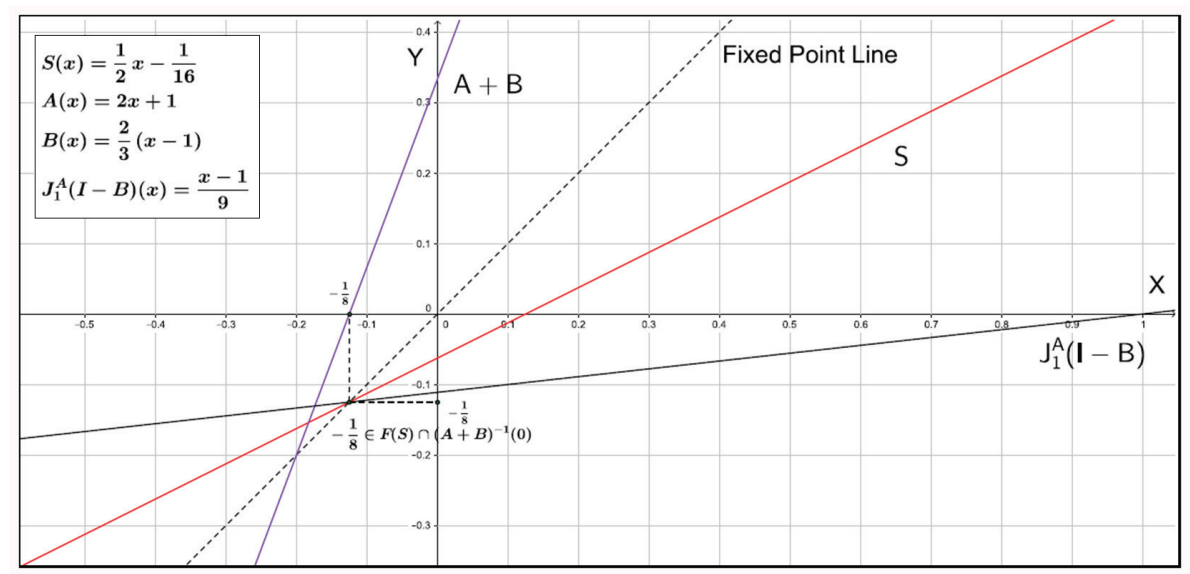

Figure 1: The solution point of $\operatorname{Fix}(S) \cap(A+B)^{-1}(0)$.

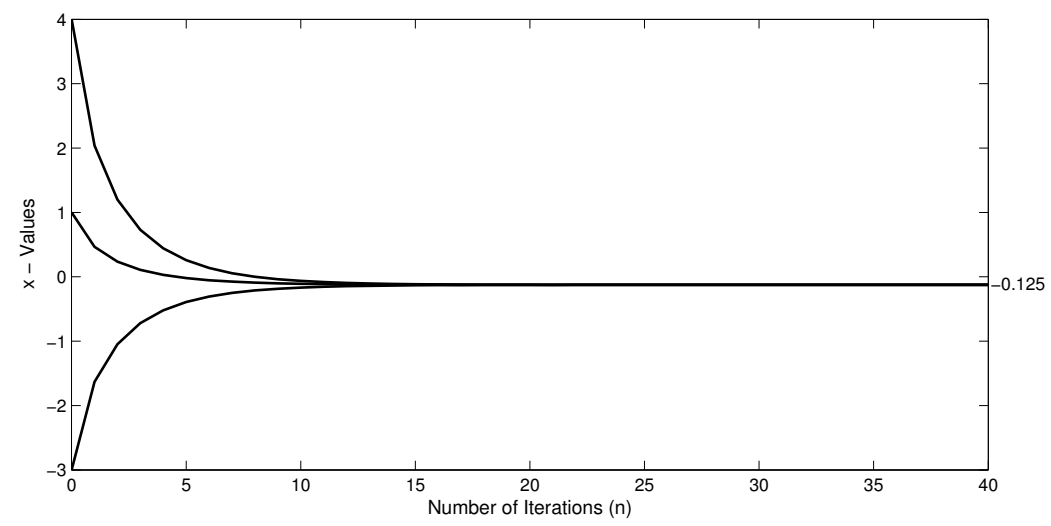

Figure 2: Behavior of $x_{n}$ for three different initial points $x_{0}=-3,1,4$.

\section{Conclusions and remarks}

Our main results extend and improve in the following:

1. Theorem 3.2 extends and improves Theorem 3.1 of Manaka and Takahashi [12, Theorem 3.1] from a Hilbert space to a Banach space and from weak convergence to strong convergence.

2. Theorem 3.2 partially extends and improves Theorem 2.1 of Cho et al. [5, Theorem 2.1] from a Hilbert space to a Banach space with uniformly convex and 2-uniformly smooth.

3. Theorem 3.2 extends and improves Theorem 3.1 of Qing and Cho [14, Theorem 3.1] from the problem of finding an element of $A^{-1}(0)$ to the problem of finding an element of $\operatorname{Fix}(S) \cap(A+B)^{-1}(0)$.

4. Theorem 3.2 extends and improves Theorem 3.7 of Sahu and Yao [17, Theorem 3.7] from the problem of finding an element of $A^{-1}(0)$ to the problem of finding an element of $\operatorname{Fix}(S) \cap(A+B)^{-1}(0)$.

5. Theorem 3.2 extends and improves Theorem 3.7 of López et al. [11, Theorem 3.7] from the problem of finding an element of $(A+B)^{-1}(0)$ to the problem of finding an element of $\operatorname{Fix}(S) \cap(A+B)^{-1}(0)$.

\section{Acknowledgment}

This project was partially supported by the Theoretical and Computational Science (TaCS) Center under Computational and Applied Science for Smart Innovation Cluster (CLASSIC), Faculty of Science, KMUTT. Moreover, also this work was supported by the Higher Education Research Promotion and 
National Research University Project of Thailand, Office of the Higher Education Commission (NRU59 Grant No.59000399). The third author was funded by King Mongkuts University of Technology North Bangkok. Contract no. KMUTNB-60-ART-085.

\section{References}

[1] K. Aoyama, H. Iiduka, W. Takahashi, Weak convergence of an iterative sequence for accretive operators in Banach spaces, Fixed Point Theory Appl., 2006 (2006), 13 pages. 2.6

[2] V. Barbu, Nonlinear semigroups and differential equations in Banach spaces, Translated from the Romanian, Editura Academiei Republicii Socialiste România, Bucharest; Noordhoff International Publishing, Leiden, (1976). 2.5

[3] E. Blum, W. Oettli, From optimization and variational inequalities to equilibrium problems, Math. Student, 63 (1994), 123-145. 4.3

[4] F. E. Browder, Fixed-point theorems for noncompact mappings in Hilbert space, Proc. Nat. Acad. Sci. U.S.A., 53 (1965), 1272-1276. 2.7

[5] S. Y. Cho, X.-L. Qin, L. Wang, Strong convergence of a splitting algorithm for treating monotone operators, Fixed Point Theory Appl., 2014 (2014), 15 pages. 1, 4.1, 2

[6] P. L. Combettes, S. A. Hirstoaga, Equilibrium programming in Hilbert spaces, J. Nonlinear Convex Anal., 6 (2005), 117-136. 4.4

[7] J.-P. Gossez, E. Lami Dazo, Some geometric properties related to the fixed point theory for nonexpansive mappings, Pacific J. Math., 40 (1972), 565-573. 2, 3

[8] S. Kitahara, W. Takahashi, Image recovery by convex combinations of sunny nonexpansive retractions, Topol. Methods Nonlinear Anal., 2 (1993), 333-342. 2.2

[9] N. Lehdili, A. Moudafi, Combining the proximal algorithm and Tikhonov regularization, Optimization, 37 (1996), 239 252. 1,1

[10] L.-S. Liu, Ishikawa-type and Mann-type iterative processes with errors for constructing solutions of nonlinear equations involving m-accretive operators in Banach spaces, Nonlinear Anal., 34 (1998), 307-317. 2.9

[11] G. López, V. Martín-Márquez, F.-H. Wang, H.-K. Xu, Forward-backward splitting methods for accretive operators in Banach spaces, Abstr. Appl. Anal., 2012 (2012), 25 pages. 1, 1, 2.4, 5

[12] H. Manaka, W. Takahashi, Weak convergence theorems for maximal monotone operators with nonspreading mappings in a Hilbert space, Cubo, 13 (2011), 11-24. 1, 1, 1

[13] B. Martinet, Régularisation d'inéquations variationnelles par approximations successives, (French) Rev. Française Informat. Recherche Opérationnelle, 4 (1970), 154-159. 1

[14] Y. Qin, S. Y. Cho, A regularization algorithm for zero points of accretive operators, Fixed Point Theory Appl., 2013 (2013), 9 pages. 3

[15] S. Reich, Weak convergence theorems for nonexpansive mappings in Banach spaces, J. Math. Anal. Appl., 67 (1979), 274-276. 2.1

[16] R.T. Rockafellar, Monotone operators and the proximal point algorithm, SIAM J. Control Optimization, 14 (1976), 877-898. 1

[17] D. R. Sahu, J. C. Yao, The prox-Tikhonov regularization method for the proximal point algorithm in Banach spaces, J. Global Optim., 51 (2011), 641-65. 1, 1, 4

[18] Y.-L. Song, L.-C. Ceng, Weak and strong convergence theorems for zeroes of accretive operators in Banach spaces, J. Appl. Math., 2014 (2014), 11 pages. 2.3

[19] T. Suzuki, Strong convergence of Krasnoselskii and Mann's type sequences for one-parameter nonexpansive semigroups without Bochner integrals, J. Math. Anal. Appl., 305 (2005), 227-239. 2.8

[20] W. Takahashi, Viscosity approximation methods for resolvents of accretive operators in Banach spaces, J. Fixed Point Theory Appl., 1 (2007), 135-147. 1

[21] W. Takahashi, Introduction to nonlinear and convex analysis, Yokohama Publishers, Yokohama, (2009). 4.1

[22] S. Takahashi, W. Takahashi, M. Toyoda, Strong convergence theorems for maximal monotone operators with nonlinear mappings in Hilbert spaces, J. Optim. Theory Appl., 147 (2010), 27-41. 4.5

[23] A. N. Tikhonov, Solution of incorrectly formulated problems and the regularization method, Soviet Meth. Dokl., 4 (1963), 1035-1038. 1

[24] A. N. Tikhonov, Improper problems of optimal planning and stable methods of their solution, Soviet Meth. Dokl., 6 (1965), 1264-1267.

[25] A. N. Tikhonov, V. Y. Arsenin, Solutions of ill-posed problems, Translated from the Russian, Preface by translation editor Fritz John, Scripta Series in Mathematics, V. H. Winston \& Sons, Washington, D.C.: John Wiley \& Sons, New York-Toronto, Ont.-London, (1977). 1 